Behavioral and neural dynamics of cognitive control in the context of rumination.

Charlotte Muscarella $^{* 1,2}$, Olivier Mairesse ${ }^{1,3,4}$, Gethin Hughes ${ }^{5}$, Eva Van den Bussche ${ }^{2}$

${ }^{1}$ Department of Psychology, Vrije Universiteit Brussel, Brussels, Belgium

${ }^{2}$ Brain \& Cognition, KU Leuven, Leuven, Belgium

${ }^{3}$ Sleep Laboratory \& Unit for Chronobiology U78, Brugmann University Hospital, Brussels,

Belgium

${ }^{4}$ Department LIFE, Royal Military Academy, Brussels, Belgium

${ }^{5}$ Department of Psychology, University of Essex, Colchester, United Kingdom

*Corresponding author should be addressed to:

\title{
Charlotte Muscarella
}

Department of Psychology

Vrije Universiteit Brussel

Pleinlaan 2

1050 Brussels

Belgium

E-mail: charlotte.muscarella@vub.be 


\begin{abstract}
Rumination is a characteristic feature of several clinical disorders (e.g., major depressive disorder, insomnia disorder). Emerging evidence suggests that a reduced flexibility in the balance between proactive and reactive control might be related to trait rumination. This study aimed to investigate the proactive-reactive control balance in the context of trait rumination. In the current study, we investigated behavioral performance and event-related potentials (ERPs) while participants were performing an AXContinuous Performance Task, to evaluate whether a shift towards more reactive control (i.e., conflict monitoring and resolution) at the expense of proactive control (i.e., maintenance and updating of taskrelevant information) is associated with increased trait rumination. Our behavioral results as well as our ERP results did not demonstrate that a shift towards more reactive control at the expense of proactive control was associated with increased trait rumination. Future research is needed to investigate the proactive-reactive control balance in the context of trait rumination. This study is the first to explore the recruitment dynamics of cognitive control using behavioral as well as electrophysiological measures in the context of rumination.
\end{abstract}

Keywords: rumination, cognitive control, AX-CPT, EEG 


\section{Introduction}

Rumination is a transdiagnostic feature that is characteristic of several mental disorders, such as major depressive disorder, obsessive compulsive disorder and insomnia disorder (Harvey, Watkins, Mansell, \& Shafran, 2004). Rumination is defined as repetitive, uncontrollable thinking concerning negative past events and/or the focus on the causes, consequences and meaning of negative mood (Nolen-Hoeksema, Wisco, \& Lyubomirsky, 2008). Rumination has negative affective consequences on mental wellbeing. For example, longitudinal studies have consistently shown that trait rumination (i.e., a higher tendency to ruminate) predicts new onsets of depressive symptomatology in initially nondepressed individuals (for a review see, Nolen-Hoeksema et al., 2008). Moreover, numerous studies have demonstrated that trait rumination is also related to the severity of depressive episodes and to recovery from depression (for a review see, Nolen-Hoeksema et al., 2008).

However, it remains unclear why some individuals seem to develop persistent rumination, whereas others succeed in disengaging from these negative thoughts. The exact cognitive mechanisms underlying rumination are still poorly understood. Although the negative content of rumination plays a considerable role in its maintenance (i.e., activation of negative mood-congruent contents in working memory (WM) by a negative event or mood will amplify and maintain persistent rumination; Siemer, 2005), there is converging evidence that impairments in cognitive control might be related to the tendency to ruminate (e.g., Joormann, 2010; Joormann \& D’Avanzato, 2010; Koster, De Lissnyder, Derakshan, \& De Raedt, 2011; Watkins, 2008). Cognitive control refers to a variety of cognitive processes, such as selective attention, context updating and response inhibition. Together, these processes coordinate our thoughts and behavior in accordance with our internal goals (Botvinick, Braver, Barch, Carter, \& Cohen, 2001; Miller \& Cohen, 2001). Cognitive control is exerted whenever people have to focus on a task. We achieve this, for instance, by actively maintaining task-relevant information in WM, by preventing taskirrelevant information to enter WM and by disengaging from information in WM that is no longer relevant for the task at hand (Botvinick, et al., 2001; Miller \& Cohen, 2001; Miyake, Friedman, Emerson, Witzki, Howerter, \& Wager, 2000). 
Watkins's Elaborated Control theory (Watkins, 2008) proposes that impairments in cognitive control will result in difficulties in effectively regulating current thoughts in response to situational demands. This, in turn, will allow repetitive off-task thoughts (such as rumination) to occur and will result in poor performance on the task at hand. Within the same vein, the Impaired Disengagement Hypothesis (Koster et al., 2011) postulates that difficulties in exerting cognitive control, more precisely difficulties in disengaging attention from negative thoughts, put individuals at risk for experiencing persistent rumination. Some evidence in support of this proposed causal relationship has been recently provided by studies aimed at enhancing cognitive control in healthy individuals using a training paradigm (e.g., Cohen, Mor, \& Henik, 2015; Hoorelbeke, Koster, Demeyer, Loeys, \& Vanderhasselt, 2016). In these studies, participants showed a reduction in reported rumination levels after cognitive control training. These findings demonstrate that cognitive control is an important mechanism in driving rumination.

Several cross-sectional (e.g., Beckwé, Deroost, Koster, De Lissnyder, \& De Raedt, 2014; Davis \& Nolen-Hoeksema, 2000; Joormann \& Gotlib, 2008) as well as prospective studies (e.g., Demeyer, De Lissnyder, Koster, \& De Raedt, 2012; Zetsche \& Joormann, 2011) in dysphoric and non-dysphoric individuals and in individuals with major depressive disorder also show that impairments in cognitive control are associated with a higher tendency to ruminate (for reviews see Whitmer, Gotlib, \& 2013; Yang, Cao, Shields, Teng, \& Liu, 2017; Zetsche, Bürkner, \& Schulze, 2018). Specifically, deficits in switching between internal representations in WM (e.g., Beckwé et al., 2014), in inhibiting previously relevant information (e.g., Whitmer \& Banich, 2007) and in updating information in WM (e.g., Joormann \& Gotlib, 2008) have all been related to trait rumination in dysphoric and non-dysphoric individuals as well as in individuals with major depressive disorder, after controlling for depressive tendencies. However, other studies have failed to find clear associations between impairments in cognitive control performance and rumination in similar samples (e.g., Alderman et al., 2015; Colzato, Steenbergen, \& Hommel, 2018), or have even found enhanced cognitive control abilities to be associated with a higher tendency to ruminate (e.g., Altamirano, Miyake, \& Whitmer, 2010; Zetsche \& Joormann, 2011). For instance, Altamirano and colleagues (2010) observed that an enhanced ability to ignore the irrelevant dimensions of a stimulus was related to higher levels of trait rumination in dysphoric and non- 
dysphoric individuals. Thus, the pattern of associations between cognitive control and trait rumination remains unclear.

However, what none of these previous studies take into account is that cognitive control can operate on different time scales, ranging from very transient to sustained across longer periods of time (Aben, Calderon, Van der Cruyssen, Picksak, Van den Bussche, \& Verguts, 2019). The Dual Mechanisms of Cognitive Control theory (DMC; Braver, 2012) offers a valuable framework for this temporal recruitment of cognitive control. This account proposes that cognitive control consists of two qualitatively distinct modes, where cognitive control is recruited either transiently or in a sustained way. According to the DMC theory, the ability to actively maintain goal-relevant information in a top-down manner reflects proactive control engagement. This sustained representation of information allows for optimal response preparation and acts in an anticipatory way to prevent conflict before it occurs. By contrast, reactive control reflects transient bottom-up goal reactivation, where cognitive control is mobilized just-in-time to detect and to resolve conflict after its onset. Although both control modes activate the same fronto-parietal brain regions, they operate on different time scales (Aben et al., 2019). Proactive control is associated with early and sustained activation in fronto-parietal areas, whereas reactive control is associated with a late and transient activation in these same regions (Aben, Calderon, Van den Bussche, \& Verguts, 2020). The DMC account presumes that both control modes have complementary costs and benefits and that successful cognition depends on the successful implementation of both (Braver, 2012). According to the DMC framework, subtle changes in situational factors (e.g., predictive value of the context information, duration of the maintenance interval, expectation of interference) will produce a change in the individual's use of a certain cognitive control strategy (Braver, Gray \& Burgess, 2007). An efficient use of cognitive control hence involves the ability to flexibly switch between control modes when needed, based on the task context, to guarantee optimal task performance. A disturbed balance in the recruitment of proactive and reactive control might result in the reliance on a control mode that is in fact not the optimal mode in a given context, leading to impairments in task performance. 
A few studies have already linked deficient proactive control to trait rumination. Beckwé and colleagues (2014) used an internal shift task where dysphoric and non-dysphoric individuals had to keep a mental count of the number of nouns versus personality traits. Participants were asked to mentally update the respective category as fast as possible. Subsequently, an internal switching cost, reflecting reduced switching abilities, was calculated by subtracting the non-switch sequences (i.e., participants had to update the same category twice in a row) from the switch sequences (i.e., participants had to switch from one category to the other). The results revealed that a higher internal switching cost was associated with higher levels of trait rumination, after controlling for depression. Similarly, difficulties in disengaging from no-longer relevant information while updating the content of WM has also been associated with trait rumination in individuals with major depressive disorder, after controlling for levels of depression (e.g., Joormann \& Gotlib, 2008; Yoon, Lemoult, \& Joormann, 2014; Zetsche, D’ Avanzato, \& Joormann, 2012). In addition, a recent meta-analysis also indicates that difficulties in discarding no longer relevant material from WM is related to trait rumination, even after controlling for levels of psychopathology (for a review, see Zetsche et al., 2018). These cognitive processes of monitoring and updating WM content by replacing no-longer-relevant information with new, more relevant information contributes to optimal proactive control engagement: when proactive control is engaged, context information should be properly represented, maintained and also updated in case this information is no longer relevant for the present context (Braver \& Cohen, 2000; 2001; Braver, Paxton, Locke, \& Barch, 2009).

In contrast, some studies in dysphoric and non-dysphoric individuals have linked enhanced reactive control to increased levels of trait rumination (Altamirano et al., 2010; Zetsche \& Joormann, 2011). Zetsche and Joormann (2011), for example, used an emotional flanker task where participants had to indicate the valence of a target word which was flanked by three distractor words. In the incongruent condition, the valence of the distractor words conflicted with the valence of the target word (e.g., negative distractors surrounding a positive target word). In the neutral condition, the valence of the distractor words was neutral compared to the valence of the target word (e.g., neutral distractors surrounding a positive target word). They found that being able to ignore negative information (i.e., negative distractor words) better compared to neutral information (i.e., neutral distractor words) 
predicted increased levels of trait rumination, after controlling for depressive symptomatology. In line with this study, participants performed a Stroop task, where they had to name the ink color of a presented word while ignoring the meaning of the word itself. Participants had to respond to congruent (i.e., ink color matches meaning of color word) and incongruent trials (i.e., ink color does not match meaning of color word). They observed that accuracy on incongruent trials (requiring increased reactive control) was positively correlated with trait rumination, after controlling for levels of depression (Altamirano et al., 2010). However, other studies did not observe a relationship between increased reactive control (as reflected in performance enhancements) and increased levels of trait rumination in dysphoric and nondysphoric individuals as well as in individuals with major depressive disorder (Alderman et al., 2015; Colzato et al., 2018). For example, Colzato et al. (2018) found that in dysphoric and non-dysphoric individuals, the size of the Stroop effect, suggesting increased reactive control, was positively related to the tendency to ruminate. However, controlling for levels of depression eliminated this significant relationship between increased reactive control and trait rumination. Still, contrary to the more unequivocal findings related to proactive control, it remains unclear whether enhanced reactive control is indeed related to trait rumination when controlling for levels of depression.

Taken together, the DMC proposes that healthy cognition relies on the flexible balance in the deployment of both cognitive control modes (Braver, 2012). The abovementioned findings suggest that a decreased proactive control, potentially accompanied by a shift towards more reactive control, might be related to trait rumination. Interestingly, the DMC framework proposes that affect-related traits can impact which cognitive control mode is primarily used (Braver, 2012). Based on the DMC perspective, the shift towards reactive control observed in several populations (e.g., in older adults, see for example Braver et al., 2009) might actually represent a compensatory recruitment to account for a reduced or deficient proactive control engagement. Specifically, the expected relationship between trait rumination and reactive control might reflect such a tradeoff between proactive and reactive control: when the capacity to actively maintain task goals in working memory is reduced, one will switch to another, less resource demanding, control strategy to optimize and preserve behavioral performance as much as possible. 
To date, all studies have investigated these two control modes in isolation, prohibiting the opportunity to study the relationship between the actual proactive-reactive control balance, as stressed by the DMC account, and rumination. No study has concurrently investigated both cognitive control modes using a single paradigm in the context of rumination. Therefore, in the current paper we propose to investigate how the balance between reactive and proactive control behaves in the context of rumination and whether a disturbance in this balance is associated with trait rumination. To this end, we will examine behavioral performance on a modified version of the AX - Continuous Performance Task (AX-CPT; Rosvold, Mirsky, Sarason, Bransome, \& Beck, 1956; Servan-Schreiber, Cohen, \& Steingard, 1996). This task has already been used extensively to assess the balance between reactive and proactive control (see for example Braver \& Barch, 2002; Braver et al., 2009; Braver, 2012). It will allow us to measure participants' reliance on both control modes by assessing 1) their ability to maintain and update contextual cue information in order to facilitate or suppress responses to a subsequent probe stimulus (i.e., proactive control) and 2) their ability to suppress an incorrect prepotent response tendency caused by contextual cue information (i.e., reactive control). Healthy young individuals are expected to primarily adopt a proactive control mode when performing the AX-CPT (Braver et al., 2009; Edwards, Barch, \& Braver, 2010; Paxton, Barch, Racine, \& Braver, 2008). Based on previous studies discussed above, we hypothesize that a disturbed proactive-reactive control balance might be related to trait rumination. Specifically, we predict that a shift from proactive control towards more reactive control is associated with increased trait rumination. Importantly, the relationship between cognitive control and rumination has mainly been investigated in relation to depression. Investigating the association between cognitive control and trait rumination in a sample of individuals without depressive symptoms will allow us to study the singular effect of cognitive control on trait rumination, without the interference of a depressive symptomatology.

\section{EXPERIMENT 1}

\section{Materials and Methods}




\section{Participants}

Ninety undergraduate students participated in this experiment. Seven participants were excluded from the analysis because of data corruption due to a software failure in the middle of the experiment. We controlled for depressive symptomology, based on the available cut-offs of the Beck Depression Inventory-II Dutch (BDI-II-NL) (Beck, Steer, \& Brown, 1996; Van der Does, 2002) by excluding dysphoric participants with BDI-II-NL scores $\geq 14$ (for a similar approach, see also e.g., De Lissnyder, Koster, Goubert, Onraedt, Vanderhasselt, \& De Raedt, 2012). Participants were naive to the purpose of the experiment. A general report describing the aims and the group-level results of the study was sent to the participants after completion of the study. Written informed consent was obtained from each participant after the experimental task had been explained and prior to the experimental session. Undergraduate students received course credits for their participation. The study protocol was approved by the ethical board of the Vrije Universiteit Brussel, Brussel, Belgium (reference 2014/234).

\section{Self-report questionnaires}

The Ruminative Response Scale (RRS-NL-EXT; Treynor, Gonzalez, \& Nolen-Hoeksema, 2003; Raes, Schoofs, Hoes, Hermans, Van Den Eede, \& Franck, 2009) was used to measure the tendency to ruminate as a reaction to feelings of sadness or depression. The RRS-NL-EXT consists of 26 items and each item is rated on a 4-point scale ('almost never' to 'almost always'). The RRS-NL-EXT exhibits adequate psychometric properties (Schoofs, Hermans, \& Raes, 2010).

The Beck Depression Inventory-II Dutch Version (BDI-II-NL; Beck et al., 1996; Van der Does, 2002) was used to measure depressive symptomatology. The BDI-II-NL is a 21-item inventory measuring depressive symptoms during the two weeks prior to participation. The BDI-II-NL has proven to have good psychometric properties (Van der Does, 2002). 


\section{Experimental task: $A X-C P T$}

Participants were seated in a quiet, dimly lit room for the duration of the experimental session. The AX-CPT was performed on a 17 -inch color LCD monitor connected to a computer running a Windows operating system. Stimulus delivery and the recording of behavioral data (reaction time and accuracy) were controlled by E-prime (www.pstnet.com; Psychology Software Tools). In the AX-CPT, performance depends on the ability to maintain and update contextual (cue) information in order to facilitate or suppress responses to a subsequent target (probe) stimulus. Participants are presented with a sequence of letters (cue-probe pairs) and are required to make a target response when an A-cue is followed by an X-probe (i.e., AX-trials) and to respond with a non-target response for all other cueprobe pairs (i.e., AY-trials, BX-trials, BY-trials). Unknown to the participants, target trials (i.e., AXtrials) occur with a high frequency, inducing the preparation of a target response whenever an A-cue is presented. Consequently, increased use of proactive control (i.e., maintenance of the task-relevant cue information to prepare a response in advance) will result in a stronger bias for a target response whenever an A-cue is encountered. In AY-trials, where the initial preparation of a target response based on the cue needs to be suppressed in order to make the correct (i.e., non-target) response, increased use of proactive control will lead to decreased performance. However, whenever a B-cue is encountered and is used proactively (i.e., facilitating the preparation of a non-target response), performance on BX-trials will be enhanced. Conversely, increased use of reactive control reflects the use of task-relevant information only when it is required (e.g., after conflict is encountered). Thus, in the current task, reactive control involves reactivating the cue information after the probe has been presented. Increased use of reactive control should thus result in poorer performance on BX-trials, since X-probes will strongly bias responses towards an incorrect target response which will have to be overcome by reactivating the nontarget response of the B-cue. However, on AY-trials increased reactive control will enhance performance since the Y-probe will prompt for a correct non-target response, without being hindered by the A-cue. In summary, the AX-CPT creates task situations where either the one or the other control strategy is detrimental for task performance, allowing us to measure participants' reliance on both proactive and reactive control. 
Participants were instructed to respond by pressing one of two color keys on an AZERTY-keyboard. Participants had to respond by pressing the green key with their left index finger when an A-cue was followed by an X-probe (i.e., target response). For all other cue-probe combinations participants had to respond by pressing the red key with their right index finger (i.e., non-target response). Participants were asked to respond as fast and as accurately as possible, but speed was emphasized. All stimuli (in 30point Courier New bold font) were presented in white on a black screen. Target trials (i.e., AX-trials) constituted $70 \%$ of the trials. Non-target trials (i.e., AY-trials, BX-trials, BY-trials) constituted $10 \%$ of the trials each. A total of 400 experimental trials were administered and equally divided over four blocks (i.e. 100 trials per block). Each block included 70 target trials and 10 of each of the non-target trials types in a random order. After each block, the participants were allowed to take a short break. The experiment started with 10 practice trials, where the trial distribution followed the same percentages as in the experimental blocks (i.e., $70 \%$ target trials, and $10 \%$ for each type of non-target trials). After each practice trial participants received feedback on the accuracy of their responses (i.e., the message "correct" or "incorrect"). This feedback was omitted during the subsequent experimental blocks. Each experimental trial started with the presentation of a fixation cross for $1000 \mathrm{~ms}$. Subsequently, a cue letter was presented for $500 \mathrm{~ms}$ again followed by a fixation cross for $2000 \mathrm{~ms}$. Then, a probe letter was presented for $500 \mathrm{~ms}$ followed by a blank screen which was presented until the participant made a response. The inter-trial interval (ITI) randomly varied from $1000 \mathrm{~ms}$ to $1500 \mathrm{~ms}$. Figure 1 depicts the sequence of a trial. 

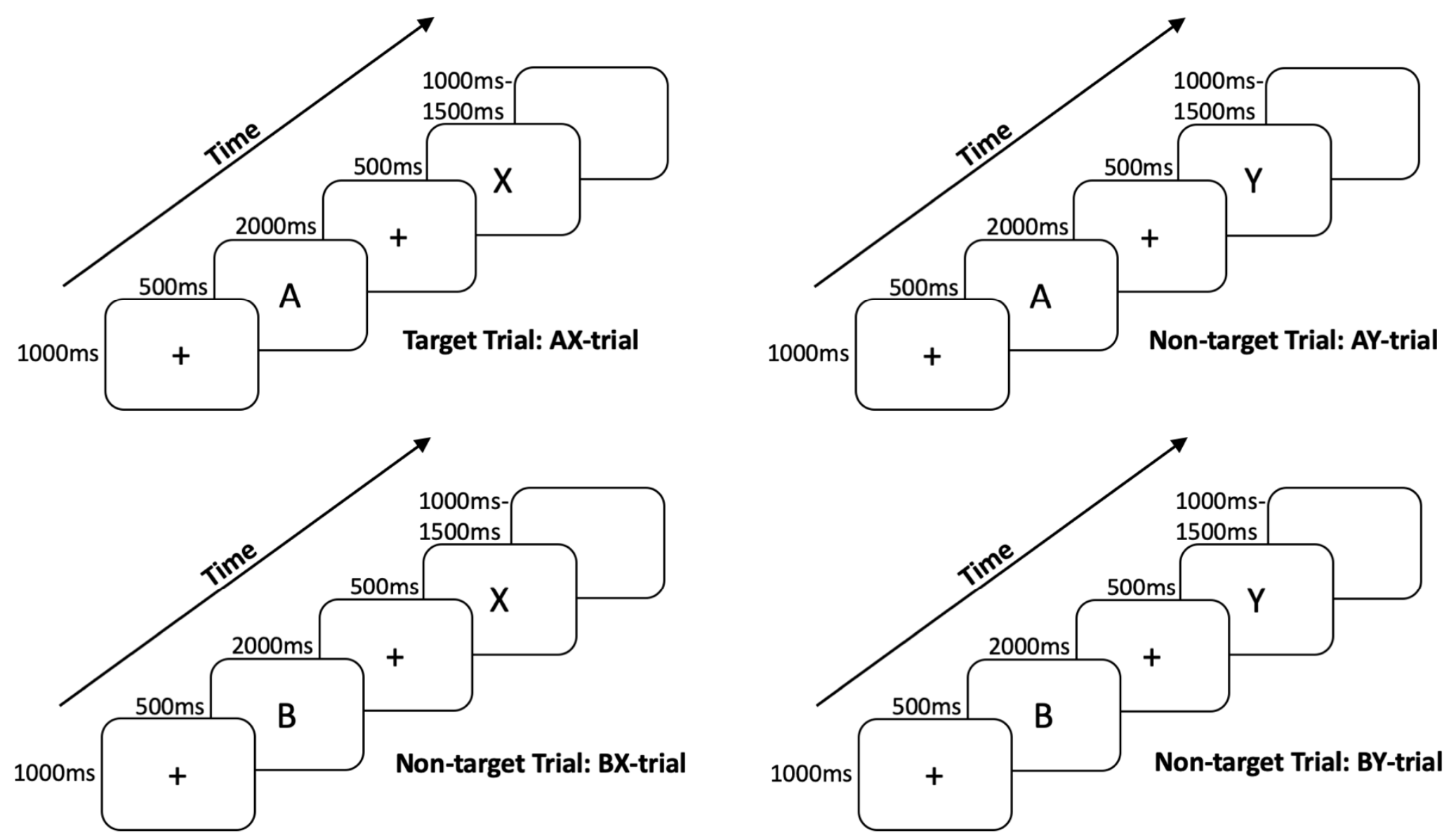

Figure 1. Examples of the different trial sequences. 


\section{Data Analysis}

All statistical analyses were conducted using IBM SPSS 25 (International Business Machines, Armonk, NY, USA). We performed two repeated measures ANCOVAs with Trial Type (4 levels: AX, $\mathrm{AY}, \mathrm{BX}, \mathrm{BY})$ as a within-subjects factor and the centered Rumination scores as a covariate on the dependent variables (i.e., median RTs of the correct responses and mean error rates). The covariate was mean centered prior to running the ANCOVA, since adding a covariate to a repeated measures analysis has been shown to bias the main effects of the within-subject factors (for an explanation of the procedure see Delaney \& Maxwell, 1981). When the assumption of homogeneity of variances was violated in the analyses, we reported the corrected values for degrees of freedom, $t$-values and $p$-values. A GreenhouseGeisser correction was applied to the $p$-values $(p \mathrm{GG})$ when the assumption of sphericity was violated. We used the Bonferroni-corrected $\alpha$-level (.05/number of comparisons) in order to determine significance of the comparisons in the pairwise samples $t$-tests in order to correct for multiple comparisons.

\section{Predictions}

Based on previous studies discussed above, we hypothesize that a disturbed proactive-reactive control balance might be related to trait rumination. Specifically, we predict a shift from proactive control towards more reactive control. The DMC theory predicts that such a shift towards reactive control relative to proactive control will be associated with enhanced performance on AY-trials: participants will prepare a correct non-target response after a Y-probe, without being hindered as much by the A-cue (i.e., a decreased maintenance of the A-cue will decrease the tendency to prepare an erroneous target response). As such, we expect that if a shift towards more reactive control at the expense of proactive control is associated with increased trait rumination, we would expect this to be associated with improved performance on AY-trials (i.e., decreased error rates and faster reaction times) (Hypothesis 1.1). Similarly, according to the DMC account, a shift towards reactive control relative to proactive control will also be associated with worse performance on BX-trials. The decreased use of 
proactive control will hamper participants in preparing an early non-target response following a B-cue. This failure to maintain the B-cue will subsequently bias them to making an incorrect target response to the X-probe. Thus, if a shift towards more reactive control instead of proactive control is associated with increased trait rumination, we expect worse performance on BX-trials (i.e., increased error rates and slower reaction times) (Hypothesis 1.2). To summarize, our primary outcome is performance (i.e., median RTs of the correct responses and mean error rates) on both AY-trials and BX-trials. We expect a shift from proactive control engagement to reactive control engagement (i.e., improved performance on AY-trials, but impaired performace on BX-trials) to be related to trait rumination.

\section{Results}

\section{Participants}

We excluded 16 participants based on a BDI-II-NL score within the clinical range. Additionally, two participants responded significantly slower $(+2 S D s)$ than the mean and four participants made significantly more errors ( $+2 S D$ s) than the mean and were therefore excluded from the analysis. Thus, the final sample consisted of 61 participants $(13 \text { men })^{1}$. Their mean age was 18.8 years $(S D=1.23$, age range 17 - 22 years). The mean BDI-II-NL score was $6.26(S D=3.57$, range 0 - 13) and the mean RRSNL-EXT score was $48.77(S D=12.22$, range 28 - 74).

\section{Behavioral results}

The median RTs of correct responses and mean error rates as a function of Trial Type (i.e., AX, AY, BX, BY) are summarized in Figure 2.

\footnotetext{
${ }^{1}$ The results remain similar when the excluded outliers were included in the analyses.
} 


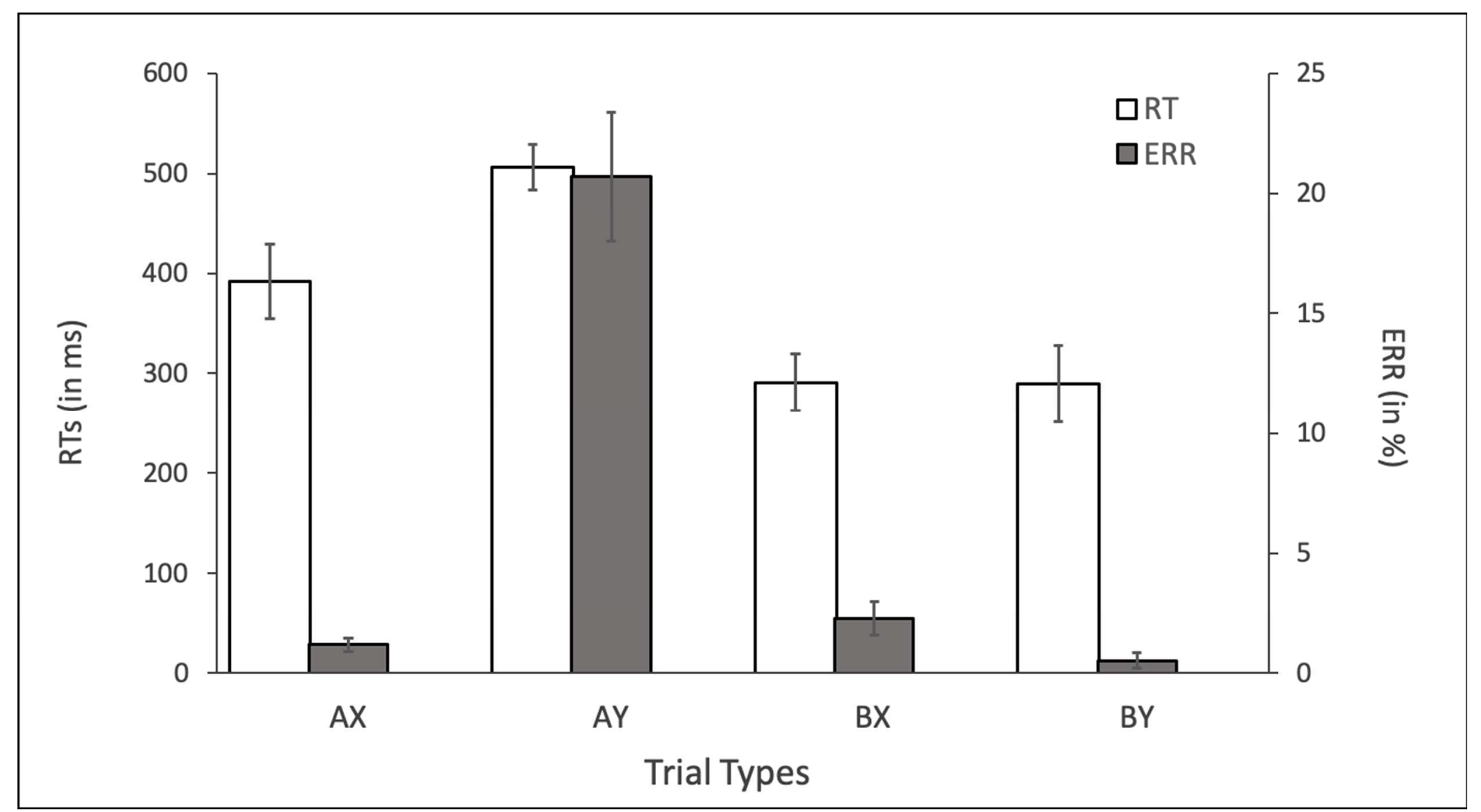

Figure 2. Means of the median reaction times (in ms) of the correct responses and the mean error rates (in \%) as a function of Trial Type (i.e., AX, AY, BX, BY) in the AX-CPT. Error bars represent confidence intervals. 


\section{Reaction times}

Inaccurate responses (i.e., target trials categorized as non-target trials or vice versa; on average 3.07 $\%$ ) were discarded for the RT analyses. A repeated measures ANCOVA with Trial Type (4 levels: AX, AY, BX, BY) as a within-subjects factor and the centered Rumination scores as a covariate on median RTs of the correct responses showed a main effect of Trial Type, $F(3,57)=221.01, p G G<.001, \eta^{2}{ }_{p}=$ .79). Post-hoc Bonferroni-corrected paired samples $t$-tests indicated that participants were slower on AY-trials $\left(M_{\mathrm{AY}}=506 \mathrm{~ms}\right)$ compared to AX-trials $\left(M_{\mathrm{AX}}=392 \mathrm{~ms}, t(60)=13.97, p<.001\right), \mathrm{BX}$-trials $\left(M_{\mathrm{BX}}=291 \mathrm{~ms}, t(60)=16.80, p<.001\right)$ and BY-trials $\left(M_{\mathrm{BY}}=290 \mathrm{~ms}, t(60)=18.61, p<.001\right)$. Posthoc Bonferroni-corrected paired samples $t$-tests indicated that participants were slower on AX-trials compared to BX-trials, $t(60)=9.76, p<.001$ and compared to BY-trials, $t(60)=10.65, p<.001)$. Moreover, post-hoc Bonferroni-corrected paired samples $t$-tests did not indicate a difference in RTs between BX-trials and BY-trials, $t(60)=0.28, p=.79$. The ANCOVA also showed a main effect of Rumination, $F(1,59)=5.01, p=.029, \eta_{p}^{2}=.078$ : the slower participants responded on all types of trials, the higher their reported levels of trait rumination were. Finally, the ANCOVA did not show an interaction between Trial Type and Rumination, $F(3,57)=1.98, p G G=.15, \eta^{2}{ }_{p}=.032$ (Hypotheses 1.1 and 1.2).

\section{Error rates}

The same repeated measures analysis conducted on the mean error rates showed a main effect of Trial Type, $\left.F(3,57)=161.89, p \mathrm{GG}<.001, \eta^{2}{ }_{p}=.73\right)$. Post-hoc Bonferroni-corrected paired samples $t$ tests indicated that participants made more errors on AY-trials $\left(M_{\mathrm{AY}}=19.02 \%\right)$ compared to AX-trials $\left(M_{\mathrm{AX}}=1.07 \%, t(60)=13.40, p<.001\right), \mathrm{BX}$-trials $\left(M_{\mathrm{BX}}=2.05 \%, t(60)=12.02, p<.001\right)$ and BY-trials $\left(M_{\mathrm{BY}}=0.46 \%, t(60)=13.55, p<.001\right)$. Post-hoc Bonferroni-corrected paired samples $t$-tests indicated that participants also made significantly more errors on BX-trials compared to AX-trials, $t(60)=2.96$, $p<.001$ and BY-trials, $t(60)=4.74 p<.001$. Finally, post-hoc Bonferroni-corrected paired samples $t$ tests indicated that participants made significantly more errors on AX-trials compared to BY-trials, $t(60)$ 
$=3.52, p<.001)$. The ANCOVA did not show a main effect of Rumination, $F(1,59)=0.92, p=.34$, $\eta_{p}^{2}=.015$, nor an interaction between Trial Type and Rumination (Hypotheses 1.1 and 1.2$), F(3,57)=$ $0.97, p G G=.34, \eta_{p}^{2}=.016$.

\section{EXPERIMENT 2}

Unexpectedly, we did not observe any shift in the balance of proactive and reactive control related to trait rumination in Experiment 1. Specifically, no performance impairments or enhancements on specific types of trials, were associated with increased trait rumination (Hypothesis 1.1 and Hypothesis 1.2). This failure to find a clear association between rumination and shifts in the balance of these different control modes might be due to the fact that it has proven to be difficult to objectively measure reactive and proactive control engagement when solely relying on behavioral measures (e.g., Krompinger \& Simons, 2011; Muscarella, Mairesse, Hughes, Neu, \& Van den Bussche, 2019). It therefore seems crucial to turn to more sensitive measures to assess the reactive-proactive control balance. For example, Krompinger and Simons (2011) asked a sample of participants with low or high depression scores to perform a Stroop task while measuring their N450. The frontal N450 component (peaking around $450 \mathrm{~ms}$ after stimulus presentation), will show more negative activation following incongruent trials than following congruent trials and is related to conflict detection (West \& Alain, 2000), and thus to reactive control in the context of Stroop tasks. The N450 likely represents activity in the anterior cingulate cortex (ACC; Hanslmayr, Pastötter, Bäuml, Gruber, Wimber, \& Klimesch, 2008; Liotti, Woldorff, Perez, \& Mayberg, 2000). Although the behavioral Stroop effect (i.e., faster reaction times in response to congruent trials compared to incongruent trials) was not related to trait rumination, they did observe that the magnitude of the N450 Stroop effect (i.e., larger N450 for incongruent compared to congruent trials), was positively related to trait rumination, but only in individuals with high depression scores. This study implies that hyperactivation of task-related brain areas might be necessary to perform cognitive control tasks at normative levels (leading to preserved behavioral performance) in individuals who are prone to ruminate. Based on this, the lack of a behavioral 
association between a shifted cognitive control balance and trait rumination in Experiment 1 might be due to compensatory brain activity in ruminators.

In a second experiment, we therefore additionally used electroencephalography (EEG) to study the temporal recruitment of proactive and reactive cognitive control and its relationship to trait rumination. Due to its superior temporal resolution, EEG will allow us to precisely measure participants' reliance on both cognitive control modes. Previous event-related potential (ERP) studies using the AX-CPT have reliably identified ERPs that are modulated by the recruitment of proactive and reactive control (e.g., Morales, Yudes, Gómez-Ariza, \& Bajo, 2015; Muscarella, et al., 2019; van Wouwe, et al., 2011).

Proactive control can be studied by means of the $\mathrm{P} 3 \mathrm{~b}$ and contingent negative variation $(\mathrm{CNV})$ ERP components, measured during the cue-probe interval. The centro-parietal P3b component (peaking around 300-600 ms after cue presentation) is thought to reflect target categorization, context updating and maintenance of task-relevant information (Donchin \& Coles, 1988; Polich, 2007; Polich \& Comerchero, 2003). Moreover, the P3b amplitude increases with the presentation of novel task-relevant information (Knight, 1984). Previous studies have already observed larger P3b amplitude for B-cues compared to A-cues, given that B-cues are presented with a lower frequency than A-cues and thus reflect a higher novelty (Morales et al., 2015; Muscarella, et al., 2019). Subsequently, the CNV, a slow cortical potential that announces the preparation of a motor response emerges (Walter, Cooper, Aldridge, Mccallum, \& Winter, 1964). The CNV appears after a warning stimulus (e.g., a cue) and announces the preparation of a motor action to a subsequent stimulus (e.g., a probe). Larger CNV amplitudes are expected whenever a response is proactively prepared (e.g., van Wouwe et al., 2011). Previous studies have observed larger $\mathrm{CNV}$ amplitudes in response to B-cues as compared to A-cues, given that B-cues accurately predict which response to make (100\% probability for a non-target response) compared to A-cues (e.g., $87.5 \%$ probability for a target response). This cue-dependent modulation of the P3b and the CNV component respectively reflects that context-relevant novel information has been appropriately updated and maintained and that adequate response preparation processes are operative, again reflecting increased proactive control (e.g., Muscarella et al., 2019). Reactive control can be indexed by several ERP components following the presentation of the probe. A fronto-central $\mathrm{N} 2$ component (peaking around 200-300 ms after probe presentation), correlates with the activation of the anterior cingulate 
cortex (ACC), a structure that is associated with response conflict detection (i.e., detection of a response conflict as triggered by the processing of irrelevant stimulus information) (Van Veen \& Carter, 2002). Following the N2, a later P3a component occurs around 300-600 ms post-probe, indexing stimulus evaluation (Coles, Gratton, Bashore, Eriksen, \& Donchin, 1985; Polich 2007; Purmann, Badde, LunaRodriguez, \& Wendt, 2011) and response inhibition (Nee, Wager, \& Jonides, 2007; Smith, Johnstone, \& Barry, 2008; Schupp, Lutzenberger, Rau, \& Birbaumer, 1994). The evaluative P3a is associated with attentional processes needed to overcome robust response tendencies (for a review, see Polich, 2007). Previous studies observed larger N2 and P3a amplitudes following AY-trials compared with the other types of trials (Beste, Domschke, Radenz, Falkenstein, \& Konrad, 2011; Morales et al., 2015; Muscarella et al., 2019). Participants will prepare a target response after presentation of the A-cue, which will conflict with the subsequent Y-probe information. Consequently, participants will detect response conflict (since cue and probe activate different response categories) and will have to resolve this response conflict by inhibiting the (incorrectly) prepared target response. Increased reactive control will be reflected in larger $\mathrm{N} 2$ and P3a amplitudes indicating increased response conflict detection and response conflict resolution.

We predict that these ERP components, indexing proactive and reactive control, will be modulated by trait rumination. Integrating EEG in our experimental design of Experiment 2, will allow us to expose the temporal dynamics of neural recruitment related to different aspects of cognitive control in the context of rumination.

\section{Materials and Methods}

\section{Participants}

Forty-two participants (i.e., volunteers and undergraduate students) participated in the experiment. All participants were right-handed, native Dutch speakers with normal or corrected-to-normal vision and had no history of epilepsy. Individuals taking psychoactive drugs were excluded from participating. Similarly, as in Experiment 1, we controlled for depressive symptomology. Participants were naive to 
the purpose of the experiment. A general report describing the aims and the group-level results of the study was sent to the participants after completion of the study. Written informed consent was obtained from each participant after the experimental task and the EEG recording procedure had been explained prior to the experimental session. Undergraduate students received course credits for their participation. The experiment was approved by the Social and Societal Ethics Committee of the Faculty of Psychology and Educational Sciences of the University of Leuven (reference: G-2015 02 187).

\section{Measures and material}

\section{Self-report questionnaires}

The self-report questionnaires used in the current experiment were identical to Experiment 1.

\section{Experimental EEG task: $A X-C P T$}

The current stimuli and procedure were highly similar to the experimental task used in Experiment 1. However, a few changes were made. Participants were seated in an electrically shielded, dimly lit room for the duration of the experimental EEG session. The AX-CPT was performed on a 15 -inch color CRT monitor. Participants had to make a target response by pressing the yellow key with their left index finger and had to make a non-target response by pressing the blue key with their right index finger. We noticed that the majority of previous studies used multiple letters (e.g., Beste at al., 2011, Braver et al., 2001, Cohen et al., 1999, Lamm et al., 2013, Lesh et al., 2013, Morales et al., 2015, van Wouwe et al., 2011) instead of only "A", "X", "B" and "Y". For consistency, "B" cues could therefore now be any letter of the alphabet, except "X", "K", and "Y" (to avoid perceptual similarity with "X"). "Y" probes could now be any letter of the alphabet except for "A" and "K". All stimuli were presented in black on a white screen. The experiment started with 10 practice trials (50\% target and $50 \%$ non-target trials) where the participants received feedback on the accuracy of their responses (i.e., the message "correct" or "incorrect"). This feedback was omitted during the subsequent experimental blocks. 


\section{EEG data recording and pre-processing}

EEG data were recorded from 64 scalp locations (BioSemi ActiveTwo System, BioSemi, Amsterdam, The Netherlands) with a sample rate of $2048 \mathrm{~Hz}$. Eye movements were recorded with electrode pairs placed $1 \mathrm{~cm}$ above and below the eye (vertical EOG) and from the outer canthi of each eye (horizontal EOG). After recording, the EEG was down-sampled offline to a $512 \mathrm{~Hz}$ sample rate. For the pre-processing of the cue-locked activity, recordings were epoched from $-500 \mathrm{~ms}$ to $+2500 \mathrm{~ms}$ relative to the onset of the cue. For the pre-processing of probe-locked activity, recordings were epoched from $-500 \mathrm{~ms}$ to $+2000 \mathrm{~ms}$ relative to the onset of the probe. Baseline correction was performed on the $200 \mathrm{~ms}$ prior to cue and probe onset, respectively. Artefact rejection was conducted by visual inspection. Artefacts not corresponding to eye blinks were manually removed. Next, independent component analysis (ICA) using MatLab (The Mathworks, Natick, MA, USA) EEGlab toolbox. Subsequently, blink components and oculomotor artefacts were manually identified, based on visual inspection and were then removed from the EEG data. Noisy channels were replaced by an interpolated weighted average from surrounding electrodes using the MatLab (The Mathworks, Natick, MA, USA) EEGlab toolbox (Delorme \& Makeig, 2004). Data from five participants (cue-locked pre-processing) and seven participants (probe-locked pre-processing) contained noisy channels. For the cue-locked pre-processing, 0.15 channels on average were interpolated. For the probe-locked pre-processing, 0.21 channels on average were interpolated. Subsequently, trials with artefacts (voltage exceeding $\pm 200 \mu \mathrm{V}$ relative to baseline, at any electrode) were removed using extreme value rejection. Finally, segments containing further artefacts, identified by visual inspection, were removed prior to averaging. Before averaging ERPs, the signals were re-referenced to the average of all 64 electrodes. When more than $25 \%$ of the trials (i.e., 100 trials) of the data of a participant still contained artefacts in the EEG signal after correction for eye movements, the data of this participant was discarded from the ERP and behavioral analyses. For plotting purposes, data were filtered using a $25 \mathrm{~Hz}$ low pass filter. 


\section{Data Analysis}

Statistical analyses were highly similar to the analyses conducted in Experiment 1.

For the ERP cue-locked analyses, we performed two repeated measures ANCOVAs with Cue (2 levels: A and B) as a within-subjects factor and the centered Rumination scores as a covariate on the dependent variables (i.e., mean average voltage of correct trials during the P3b time-window and $C N V$ time-window). For the ERP probe-locked analyses, we performed two repeated measures ANCOVAs with Trial Type (4 levels: AX, AY, BX, BY) as a within-subjects factor and the centered Rumination scores as a covariate on the dependent variables (i.e., mean average voltage of correct trials during the N2 time-window and P3a time-window). For the behavioral analyses, we performed two repeated measures ANCOVAs with Trial Type (4 levels: AX, AY, BX, BY) as a within-subjects factor and the centered Rumination scores as a covariate on the dependent variables (i.e., median RTs of the correct responses and mean error rates).

\section{ERP components and analyses}

Only the experimental trials with correct responses (on average $97 \%$ for the cue-locked analyses and $98 \%$ for the probe-locked analyses) were included in the ERP analyses. To define the spatial topography and time-windows, we first averaged the waveforms for each Cue (i.e., A-cue and B-cue) and each Trial Type (i.e., AX, AY, BX, BY), respectively for the cue-locked analyses and for the probe-locked analyses. Based on the collapsed waveforms, we defined the spatial topography for the analysis of cue components based on the grand-average difference plot between B-cues and A-cues, and probe components based on a grand-average difference plot of response conflict (i.e., the difference between BY-trials and AY-trials). Based on the collapsed waveforms and on prior research studying these ERP components, we defined the time-windows for each component. Subsequent EEG analysis, data averaging and data handling were conducted using MatLab and custom-built MatLab scripts (The Mathworks, Natick, MA, USA). 
$P 3 b$. For the P3b, the EEG recording was filtered between $0.01 \mathrm{~Hz}$ (high-pass filter) and $30 \mathrm{~Hz}$ (lowpass filter), slope $24 \mathrm{~dB} /$ octave. In line with the approach used in prior research using the AX-CPT (Morales et al., 2015; van Wouwe et al., 2011), the P3b amplitude was calculated over a time-window between $400 \mathrm{~ms}$ to $700 \mathrm{~ms}$ after cue presentation, corresponding to the latencies in which the grand averages exceeded a quarter of the P3b peak amplitudes. For this analysis, we pooled EEG activity from a cluster of centro-parietal electrodes (CP1, CPz, CP2, P1, Pz, P2, PO3, POz, and PO4).

$C N V$. For the CNV, the EEG data was filtered between $0.01 \mathrm{~Hz}$ (high-pass filter) and $30 \mathrm{~Hz}$ (lowpass filter), slope $24 \mathrm{~dB} /$ octave. We examined the CNV in a time window between $1000 \mathrm{~ms}$ and 2000 ms post-cue. For this analysis, we pooled EEG activity across a region of midline electrodes ( $\mathrm{Fz}, \mathrm{FCz}$, $\mathrm{Cz}$, and $\mathrm{CPz}$ ).

N2. In order to avoid masking of the $\mathrm{N} 2$ by the larger P3a amplitudes, a $2 \mathrm{~Hz}$ (high-pass) and a 12 $\mathrm{Hz}$ (low-pass), slope $24 \mathrm{~dB} /$ octave filter was applied to filter out the P3a component (Clayson \& Larson, 2011; Morales et al., 2015; van Wouwe et al., 2011). The N2 was calculated by pooling data over the centro-parietal electrodes $\mathrm{FCz}, \mathrm{Cz}, \mathrm{CPz}$ and $\mathrm{Pz}$ in the $40 \mathrm{~ms}$ period around the peak of the component, which resulted in a time-window between $240 \mathrm{~ms}$ and $280 \mathrm{~ms}$ (based on Donchin \& Coles, 1988; Desender, Van Opstal, Hughes, \& Van den Bussche, 2016, Muscarella et al., 2019).

P3a. For the P3a, the EEG over the frontal electrode Fz was filtered at $0.01 \mathrm{~Hz}$ (high-pass) and 30 $\mathrm{Hz}$ (low-pass), slope $24 \mathrm{~dB} /$ octave. The P3a was calculated over the electrode Fz in the $80 \mathrm{~ms}$ period around the peak of the component. This resulted in a time-window between $320 \mathrm{~ms}$ and $400 \mathrm{~ms}$ (based on Paxton et al., 2008).

\section{Predictions}

\section{ERP hypotheses}

As explained above, making inferences regarding proactive and reactive control engagement based on behavioral data alone might prove difficult. However, by using EEG we can further disentangle the unique contribution of proactive control. More specifically, by investigating brain activity following the 
cue, we can determine the extent to which participants maintain this information to prepare their upcoming response (i.e., proactive control).

For the cue-related ERP components, we expect a decreased use of proactive control to be associated with trait rumination. This will be reflected in an association between smaller P3b differences between B-cues and A-cues (suggesting difficulties engaging with relevant cue information and updating nolonger relevant information with novel relevant cue information) and trait rumination (Hypothesis 2.1). If decreased proactive control is associated with trait rumination, we also expect that trait rumination will vary as a function of the difference in $\mathrm{CNV}$ between A-cues and B-cues (i.e., increasing trait rumination will be related to a smaller CNV difference between A-cues and B-cues), reflecting an impaired use of the cue information to adequately prepare for the upcoming response (Hypothesis 2.2).

For the probe-related ERP components, we expect that reactive control (which is reflected in larger $\mathrm{N} 2$ and P3a amplitudes) will be recruited on BX-trials and AY-trials, since cues and probes on these trial types activate different response categories. However, a decreased proactive control with trait rumination will not only be reflected in the cue-related ERP components but also in the probe-related components. Because of a decreased proactive control, response competition will change on BX-and AY-trials. Specifically, reactive control will have to be engaged to a lesser extent after Y-probes on AYtrials. A decreased maintenance of the A-cue, will result in a smaller response conflict between the Acue and the Y-probe, leading to smaller N2 amplitudes (Hypothesis 2.3). In addition, the Y-probe will prompt for the correct non-target response. A decreased maintenance of the A-cue will require less inhibition of a prepotent tendency to make a target response based on this cue, which will be reflected in smaller P3a amplitudes (Hypothesis 2.4). Similarly, on BX-trials, a decreased maintenance of the Bcue, will result in a smaller response conflict between the B-cue and the X-probe. However, as the Xprobe triggers an incorrect response tendency towards a target response, participants will detect a response conflict (as reflected by larger N2 amplitudes; Hypothesis 2.5) and will need to inhibit a prepotent tendency to make a target response (as reflected by larger P3a amplitudes; Hypothesis 2.6) in order to make a correct non-target response. In general, if increased reactive control is associated with trait rumination, we would expect that this will mostly be apparent in the AY-trials and BX-trials with larger N2 amplitudes and larger P3a amplitudes in response to the probe. 


\section{Behavioral hypotheses}

Similar to Experiment 1, we again hypothesize that a shift towards more reactive control at the expense of proactive control might be related to trait rumination, leading to enhanced AY-performance (Hypothesis 2.7) and decreased BX-performance (Hypothesis 2.8). In addition, we can now also determine whether a decreased performance on BX-trials and an increased performance on AY-trials results from decreased proactive control recruitment or an increased reactive control recruitment by complementing the behavioral results with the ERP results. However, in line with the results of Experiment 1, it could also be that we do not find cognitive control dynamics to be associated with trait rumination on a behavioral level (possibly due to compensatory mechanisms).

\section{Results}

\section{Participants}

The data of three participants were removed due to excessive noise and artifacts on the EEG recordings. Additionally, two participants responded significantly slower $(+2 S D s)$ than the mean and were therefore excluded from the analysis ${ }^{2}$. We excluded three participants based on a BDI-II-NL score within the clinical range. The EEG recording of one participant experienced technical problems (e.g., data loss of $2.75 \%$ ), so the analysis was performed on the remaining part of the data of this participant. Thus, the final sample consisted of 34 participants ( 7 men). Their mean age was 21.2 years $(S D=2.35$, age range 18 - 25 years). The mean BDI-II-NL score was $4.91(S D=3.40$, range 0 - 12$)$ and the mean score RRS-NL-EXT was $40.94(S D=10.27$, range 26 - 69).

\footnotetext{
${ }^{2}$ The results remain similar when the excluded outliers were included in the analyses.
} 


\section{ERP results}

\section{Cue locked ERPs}

$P 3 B$. Figure 3 presents the grand average cue-locked ERPs for the centro-parietal electrodes elicited by the different cues (i.e., A and B).

A repeated measures ANCOVA with Cue (2 levels: A and B) as a within-subjects factor and the mean centered Rumination scores as a covariate on the mean average voltage of correct trials during the P3b time-window (400 ms - $700 \mathrm{~ms}$ ) across centro-parietal electrodes, showed a main effect of Cue, $F(1$, $32)=115.39, p<.001, \eta^{2}=.78$. The P3b amplitude was larger for B-cues $\left(M_{\mathrm{B}-\text { cue }}=4.71 \mu \mathrm{V}\right)$ compared to that for A-cues $\left(M_{\mathrm{A}-\text { cue }}=1.53 \mu \mathrm{V}\right)$. The ANCOVA showed no interaction between Cue and Rumination, $F(1,32)=0.005, p=.95, \eta_{p}^{2}<0$ (Hypothesis 2.1$)$, nor a main effect of Rumination, $F(1$, $32)=0.18, p=.68, \eta^{2} p=.006$

$C N V$. Figure 3 presents the grand average cue-locked ERPs for the fronto-central electrodes elicited by the different cues (i.e., A and B). 

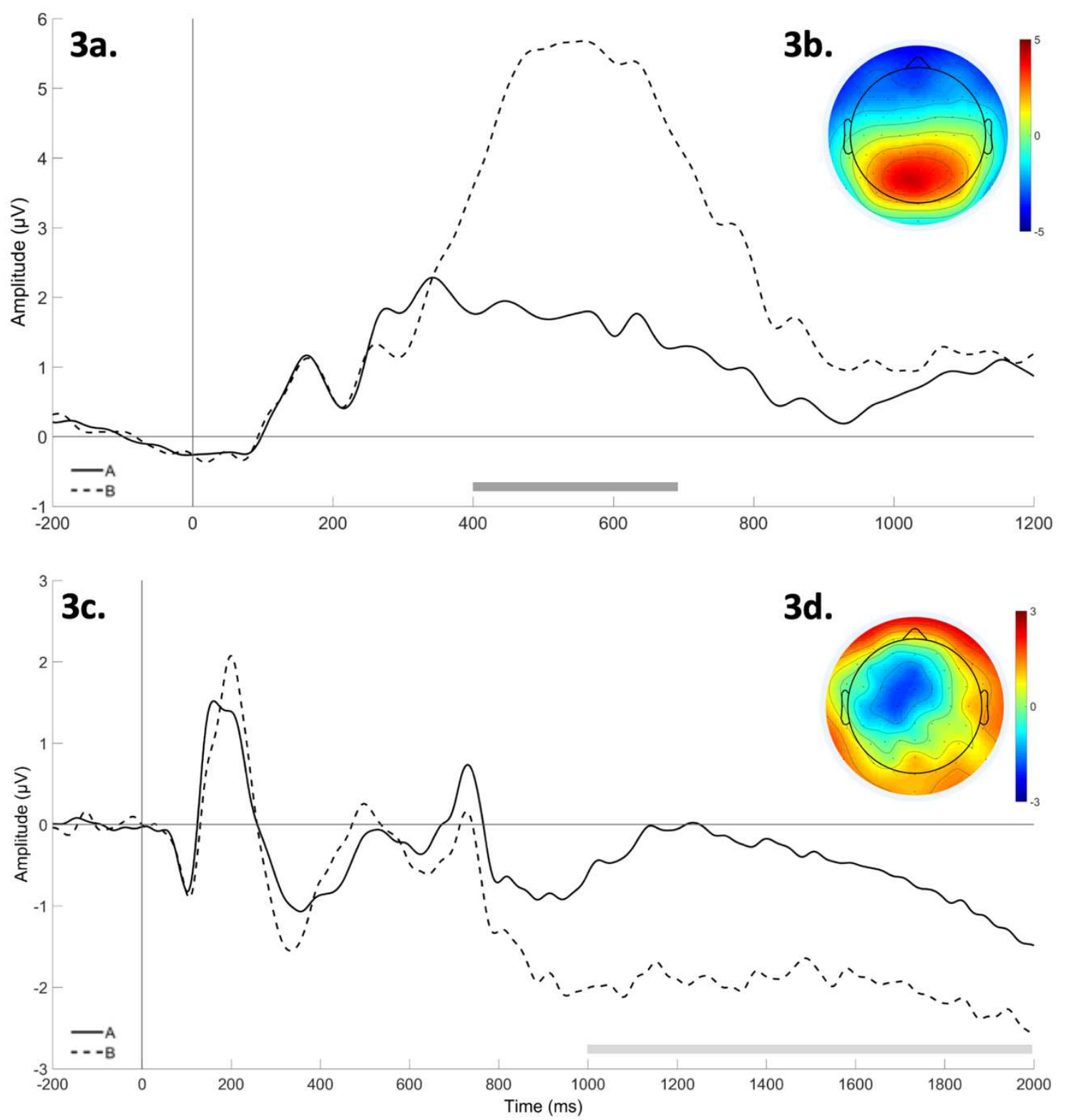

Figure 3. 3a. Grand averages of the cue-locked ERPs evoked at the centro-parietal electrodes (CP1, P1, PO3, POz, Pz, CPz, CP2, P2 and PO4). Dark gray horizontal bars indicate the P3b time-windows of analysis, respectively $400 \mathrm{~ms}$ to $700 \mathrm{~ms}$. 3b. Topographic distribution plot for the P3b effect (B - A cues) over the $400 \mathrm{~ms}$ to $700 \mathrm{~ms}$ time-window. 3c. Grand averages of the cue-locked ERPs evoked at the fronto-central electrodes $(\mathrm{Fz}, \mathrm{FCz}, \mathrm{Cz}$ and $\mathrm{CPz})$. The light grey bar indicates the $\mathrm{CNV}$ window of analysis (1000 ms to $2000 \mathrm{~ms}$ ). 3d. Topographic distribution plot for the CNV effect (B - A cues) over the $1000 \mathrm{~ms}$ to $2000 \mathrm{~ms}$ time-window. 
A repeated measures ANCOVA with Cue (2 levels: A and B) as a within-subjects factor and the centered Rumination scores as a covariate on the mean average voltage of correct trials during the $C N V$ time-window (1000 ms - $2000 \mathrm{~ms})$ across the fronto-central electrodes showed a main effect of Cue, $F(1,32)=39.27, p<.001, \eta_{p}^{2}=.55$. The CNV amplitude was larger for B-cues $\left(M_{\mathrm{B}-\text { cue }}=-1.83 \mu \mathrm{V}\right)$ compared to A-cues $\left(M_{\mathrm{A}-\mathrm{cue}}=-0.48 \mu \mathrm{V}\right)$. The ANCOVA did not show a main effect of Rumination, $F(1$, $32)=2.30, p=.14, \eta_{p}^{2}=.067$, nor an interaction between Cue and Rumination, $F(1,32)=0.30, p=.59$, $\eta_{p}^{2}=.009$ (Hypothesis 2.2).

\section{Probe locked ERPs}

N2. Figure 4 presents the grand average probe-locked ERPs for the centro-parietal electrodes elicited by the different trial types (i.e., AX, AY, BX, BY).

A repeated measures ANCOVA with Trial Type (4 levels: AX, AY, BX, BY) as a within-subjects factor and the centered Rumination scores as a covariate on the mean average voltage of correct trials during the $N 2$ time-window (240 ms - $280 \mathrm{~ms}$ ) across the centro-parietal electrodes showed a main effect of Trial Type, $F(3,30)=73.09, p G G<.001, \eta^{2}=.70$. Post-hoc Bonferroni-corrected paired samples $t$ tests indicated that the $\mathrm{N} 2$ amplitude for AY-trials $\left(M_{\mathrm{AY}}=-2.40 \mu \mathrm{V}\right)$ was larger than that for AX-trials $\left(M_{\mathrm{AX}}=-0.28 \mu \mathrm{V}, t(33)=-9.52, p<.001\right), \mathrm{BX}$-trials $\left(M_{\mathrm{BX}}=0.17 \mu \mathrm{V}, t(33)=-11.57, p<.001\right)$ and $\mathrm{BY}-$ trials $\left(M_{\mathrm{BY}}=-0.15 \mu \mathrm{V}, t(33)=-11.29, p<.001\right)$. Post-hoc Bonferroni-corrected paired samples $t$-tests indicated that the $\mathrm{N} 2$ amplitude for BY-trials was also larger than that for BX-trials, $t(33)=-2.98, p=$ .005. No difference (using a Bonferonni corrected $\alpha$ ) was found between the N2 amplitude for AX-trials and BX-trials, $t(33)=-2.27, p=.033$ and between the $\mathrm{N} 2$ amplitude for AX-trials and BY-trials, $t(33)$ $=-0.71, p=.48$. The ANCOVA did not show a main effect of Rumination, $F(1,32)=2.88, p=.099$, $\eta^{2}{ }_{p}=.083$, nor an interaction between Trial Type and Rumination, $F(3,30)=0.87, p G G=.44, \eta_{p}^{2}=$ .026 (Hypotheses 2.3 and 2.5). 
P3a. Figure 4 presents the grand average probe-locked ERPs for the centro-parietal electrodes elicited by the different trial types (i.e., AX, AY, BX, BY).

A repeated measures ANCOVA with Trial Type (4 levels: AX, AY, BX, BY) as a within-subjects factor and the centered Rumination scores as a covariate on the mean average voltage of correct trials during the P3a time-window (320 ms - $400 \mathrm{~ms}$ ) across the frontal electrode showed a main effect of Trial Type, $F(3,30)=21.01, p G G<.001, \eta_{p}^{2}=.40$. Post-hoc Bonferroni-corrected paired samples $t$ tests indicated that the P3a amplitude for AY-trials $\left(M_{\mathrm{AY}}=0.83 \mu \mathrm{V}\right)$ was larger than that for AX-trials $\left(M_{\mathrm{AX}}=-2.61 \mu \mathrm{V}, t(33)=5.39, p<.001\right), \mathrm{BX}$-trials $\left(M_{\mathrm{BX}}=-2.88 \mu \mathrm{V}, t(33)=4.76, p<.001\right)$ and $\mathrm{BY}-$ trials $\left(M_{\mathrm{BY}}=-2.93 \mu \mathrm{V}, t(33)=5.62, p<.001\right)$. Post-hoc Bonferroni-corrected paired samples $t$-tests did not show a significant difference between the P3a amplitude for AX-trials and BX-trials, $t(33)=0.69$, $p=.50, \mathrm{AX}$-trials and BY-trials, $t(33)=0.84, p=.41$, and BX-trials and BY-trials, $t(33)=0.13, p=.90$. The ANCOVA did not show a main effect of Rumination, $F(1,32)=1.32, p=.26, \eta^{2}{ }_{p}=.040$, nor an interaction between Trial Type and Rumination, $F(3,30)=1.16, p G G=.32, \eta^{2}{ }_{p}=.035$ (Hypotheses 2.4 and 2.6). 

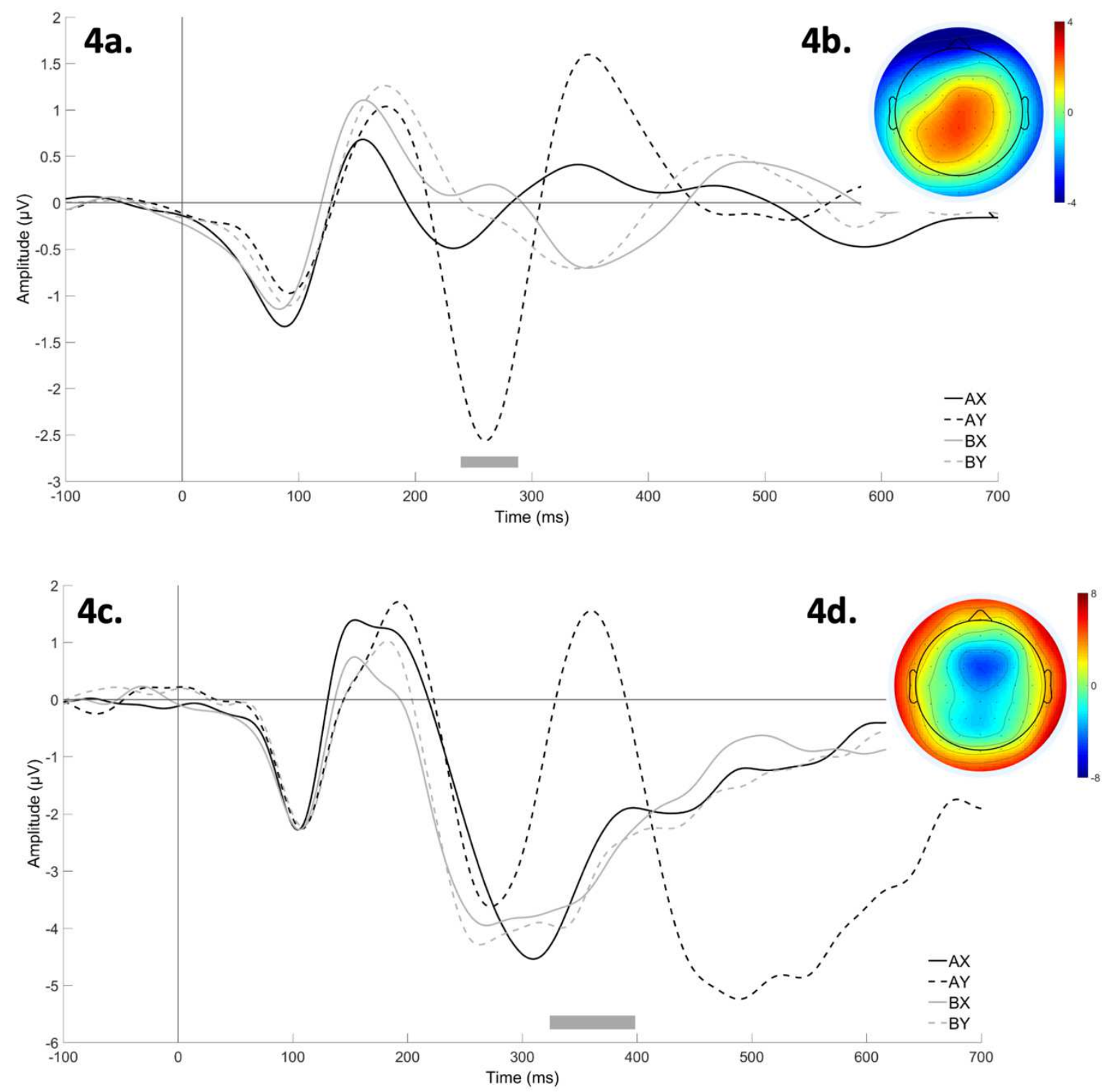

Figure 4. 4a. Grand averages of the probe-locked ERPs evoked over the centro-parietal electrodes (FCz, Cz, CPz and Pz). The grey bar indicates the N2 window of analysis (240 ms to $280 \mathrm{~ms}$ ). $4 \mathrm{~b}$. Topographic distribution plot for the N2 effect (BY - AY trials) over the $240 \mathrm{~ms}$ to $280 \mathrm{~ms}$ time-window. 4c. Grand averages of the probe-locked ERPs evoked at the frontal electrode (Fz). The grey bar indicates the P3a window of analysis ( $320 \mathrm{~ms}$ to $400 \mathrm{~ms}$ ). 4d. Topographic distribution plot for the P3a effect (BY - AY trials) over the $320 \mathrm{~ms}$ to $400 \mathrm{~ms}$ time-window. 


\section{Behavioral results}

The median RTs of correct responses and mean error rates as a function of Trial Type (i.e., AX, AY, BX, BY) are summarized in Figure 5.

\section{Reaction times}

Inaccurate responses (i.e., target trials categorized as non-target trials or vice versa; on average 3.36 \%) were discarded for the RT analyses. A repeated measures ANCOVA with Trial Type (4 levels: AX, $\mathrm{AY}, \mathrm{BX}, \mathrm{BY}$ ) as a within-subjects factor and the centered Rumination scores as a covariate on median RTs of the correct responses showed a main effect of Trial Type, $F(3,30)=211.50, p G G<.001, \eta_{p}^{2}=$ .87. Post-hoc Bonferroni-corrected paired samples $t$-tests indicated that participants were slower on AYtrials $\left(M_{\mathrm{AY}}=476 \mathrm{~ms}\right)$ compared to AX-trials $\left(M_{\mathrm{AX}}=341 \mathrm{~ms}, t(33)=16.79, p<.001\right), \mathrm{BX}$-trials $\left(M_{\mathrm{BX}}=\right.$ $278 \mathrm{~ms}, t(33)=20.27, p<.001)$ and BY-trials $\left(M_{\mathrm{BY}}=286 \mathrm{~ms}, t(33)=22.82, p<.001\right)$. Post-hoc Bonferroni-corrected paired samples $t$-tests indicated that participants were slower on AX-trials compared to BX-trials, $t(33)=5.82, p<.001$ and compared to BY-trials, $t(33)=4.91, p<.001$. Moreover, post-hoc Bonferroni-corrected paired samples $t$-tests indicated no difference in RTs between BX-trials and BY-trials, $t(33)=-1.88, p=.069$. The ANCOVA did not show a main effect of Rumination, $F(1,32)=3.65, p=.065, \eta_{p}^{2}=.10$, nor an interaction between Trial Type and Rumination, $F(3,30)=2.31, p G G=.12, \eta_{p}^{2}=.067$ (Hypotheses 2.7 and 2.8).

\section{Error rates}

The same repeated measures analysis conducted on the mean error rates showed a main effect of Trial Type, $F(3,30)=30.64, p \mathrm{GG}<.001, \eta_{p}^{2}=.49$. Post-hoc Bonferroni-corrected paired samples $t$ tests indicated that participants made more errors on AY-trials $\left(M_{\mathrm{AY}}=10.71 \%\right)$ compared to AX-trials $\left(M_{\mathrm{AX}}=1.06 \%, t(33)=5.65, p<.001\right), \mathrm{BX}$-trials $\left(M_{\mathrm{BX}}=1.11 \%, t(33)=5.42, p<.001\right)$ and BY-trials $\left(M_{\mathrm{BY}}=0.55 \%, t(33)=5.98, p<.001\right)$. Post-hoc Bonferroni-corrected paired samples $t$-tests indicated no difference in error rates between $\mathrm{AX}$-trials and $\mathrm{BX}$-trials, $t(33)=-0.15, p=.88$, between $\mathrm{BX}$-trials 
and BY-trials, $t(33)=1.64, p=.11$ and between AX-trials and BY-trials, $t(33)=1.76, p=.088$. The ANCOVA did not indicate a main effect of Rumination, $F(1,32)=0.14, p=.71, \eta^{2}{ }_{p}=.004$, nor an interaction between Trial Type and Rumination, $F(3,30)=0.36, p G G=.57, \eta^{2}{ }_{p}=.011$ (Hypotheses 2.7 and 2.8). 


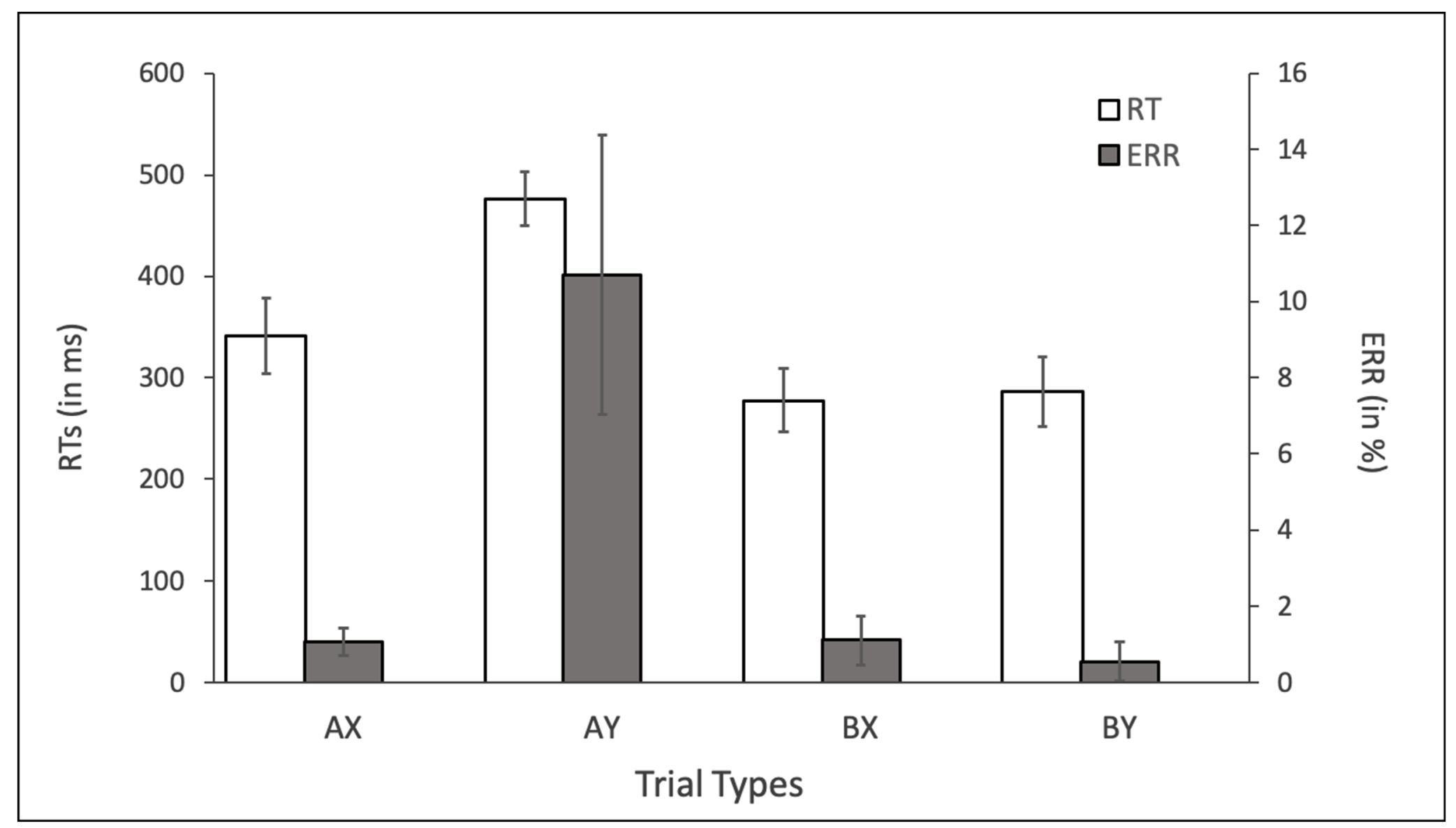

Figure 5. Means of the median reaction times (in ms) of the correct responses and the mean error rates (in \%) as a function of Trial Type (i.e., AX, AY, BX, BY) in the AX-CPT. Error bars represent confidence interval. 


\section{Discussion}

The present study is the first to investigate whether a shift towards more reactive control instead of proactive control is associated with increased trait rumination. Using an AX-CPT, we examined whether impairments in context updating and the maintenance of task-relevant information, reflecting a decreased proactive control, was associated with trait rumination. In contrast, we investigated whether enhanced response conflict detection and response inhibition, reflecting increased reactive cognitive control, were associated with trait rumination. The results of both experiments did not provide support for our hypotheses.

Consistent with previous research using the AX-CPT (e.g., Paxton et al., 2008; Morales et al., 2015), we observed the expected behavioral and neurophysiological effects. More specifically, in line with DMC predictions (Braver et al., 2009; Edwards et al., 2010; Paxton et al., 2008), we observed that participants in both experiments employed a proactive control strategy (relative to a reactive control strategy) to solve the task. More specifically, in general, participants employed a proactive control strategy relative to a reactive control strategy to perform the task, given that participants' overall performance was significantly worse on AY-trials compared to the other types of trials. This result indicates that participants relied on the cue-information to prepare a response to a subsequent probe. In the case of AY-trials, the preparation of a target response prompted by the A-cue, biased participants towards an incorrect response tendency. The use of a proactive strategy in the behavioral data was further supported by the results of the cue-related and probe-related ERP components. First, we found that the P3b was larger for B-cues compared to the P3b for A-cues, indicating that participants were attentive to the presentation of novel task-relevant information. Second, the CNV amplitude was also larger for Bcues compared to A-cues, reflecting adequate response preparation processes. Third, we observed larger $\mathrm{N} 2$ and P3a amplitudes in response to AY-trials compared to the other types of trials, indicating that participants experienced a larger response conflict for AY-trials (increased reactive control due to a well-functioning proactive control; N2) and consequently showed an increased need for inhibition of an incorrect response tendency towards a target response triggered by A-cues (P3a). 
Furthermore, in accordance with previous research (Beckwé et al., 2014), we observed in Experiment 1 that given a preserved accuracy performance, trait rumination was associated with generally slower reaction times. According to the processing efficiency theory (Eysenck \& Calvo, 1992; Eysenck, Derakshan, Santos, \& Calvo, 2007), these increased reaction times, together with preserved accuracy, might reflect compensatory enhancement of cognitive control in order to maintain task performance.

Unexpectedly, we did not observe that a shift towards more reactive control instead of proactive control was associated with trait rumination. With regards to proactive control, previous studies focusing on proactive mechanisms demonstrated an impaired proactive control in the context of rumination (e.g., Beckwé et al., 2014; Whitmer \& Banich, 2007). However, we did not find an association between attenuated cue-related P3b (Hypothesis 2.1) and CNV components (Hypothesis 2.2) (implying impaired maintenance of the cue-information and diminished response preparation processes) and trait rumination. With regards to reactive control, previous studies focusing on reactive mechanisms demonstrated an enhanced reactive control in the context of rumination (Altamirano et al., 2010; Zetsche \& Joormann, 2011). However, we did not find an association between rumination and altered proberelated N2 (Hypotheses 2.3 and 2.5) and P3a components (Hypotheses 2.4 and 2.6) (implying altered response conflict detection processes and response inhibition processes). Behaviorally, we did not find a decreased performance on BX-trials (Hypotheses 1.2 and 2.8) nor an increased performance on AYtrials (Hypotheses 1.1 and 2.7), associated with decreased proactive control and increased reactive control, to be related with trait rumination.

It could be argued that impairments in the maintenance of task-relevant information in the context of trait rumination would only show up under increased WM maintenance demands, for instance with longer cue-probe intervals than the relatively short cue-probe intervals (i.e., $2 \mathrm{~s}$ ) that were used in the current study. Specifically, under conditions where the cue-probe interval is long (e.g., $5 \mathrm{~s}$ or more), task-relevant information should not only be represented but should also be actively maintained over time (Braver \& Barch, 2002; Braver et al., 2005). Therefore, longer cue-probe intervals require stronger context maintenance (Braver \& Barch, 2002). Accordingly, impairments in context maintenance might be more apparent following longer delays. A recent study by Muscarella et al. (2019) observed that when using a longer cue-probe interval (i.e., $4900 \mathrm{~ms}$ ), individuals with insomnia disorder (which were 
characterized by increased levels of trait rumination) showed an impaired proactive control engagement (as reflected by the P3b and CNV components). Likewise, in previous research indicating proactive control impairments in the context of trait rumination (Zetsche et al., 2012) the maintenance interval applied in a WM Selection task (WMST) was approximately 9 s. Future studies examining proactive control in the context of trait rumination should therefore include longer cue-probe intervals to create a task context where a sufficient amount of proactive control has to be exerted for optimal task performance.

Moreover, it has been suggested that the involvement of proactive and reactive control is contextdependent, favoring one cognitive control strategy over the other depending on whether conflict events occur with low or high frequency (Aben et al., 2019; Jaspar, Manard, Dideberg, Bours, Maquet, \& Collette, 2016). For example, using a Stroop task, it has been shown that in a context containing mainly congruent trials, participants shift towards more reactive control, exerting control in a just-in-time manner. In contrast, in a mainly incongruent context, participants shift towards more proactive control, exerting control to prevent conflict before its occurrence (Braver et al., 2005). In our current design, we only created a mainly congruent context, where the majority (i.e., $80 \%$ ) of the trials consisted of congruent trials (i.e., AX-trials and BY-trials; where cue and probe activate the same response category) and the minority (i.e., $20 \%$ ) of trials of incongruent trials (i.e., AY-trials and BX-trials; where cue and probe activate opposite response categories). In this task context, reactive control is triggered on incongruent trials. Our results demonstrate that specifically on AY-trials, increased reactive control (as reflected by larger N2 and P3a amplitudes) was recruited. However, this shift towards reactive control was not associated with trait rumination. To examine the reactive-proactive control balance in the context of trait rumination more elaborately, future studies should also include a mostly incongruent context, which triggers proactive control.

Furthermore, it could also be argued that our version of the AX-CPT may not have been challenging enough to observe impairments in proactive control associated with trait rumination. Indeed, differences in task difficulty between our task and tasks used in previous studies observing proactive impairments might account for the failure to find clear impairments in proactive control associated with trait rumination in our study. In previous research observing proactive control impairments in the context of 
rumination (Joormann \& Gotlib, 2008; Yoon, et al., 2014; Zetsche et al., 2012), participants had to memorize a set of six items during a learning phase, and were subsequently instructed to forget three of the six items of that list. Finally, a probe item appeared, and participants had to indicate whether the probe item belonged to the final list or not. In this task, participants not only need to maintain taskrelevant information in WM, but they also need to update this information in WM when this information is no longer relevant for the present context. Specifically, participants need to update information within the current trial. In contrast, in our design, participants need to update information across different trials. A manipulation within the same trial might be more challenging and might subsequently recruit more proactive control processes. Future studies examining proactive control in the context of trait rumination should therefore include a challenging task context where task-relevant information needs to be maintained and updated within the same context.

There are some limitations to this study that should be addressed in future research. First, using this AX-CPT, it remains difficult to objectively disentangle reactive control from proactive control engagement. Specifically, making behavioral and electrophysiological inferences regarding proactive control engagement based on post-probe data remains difficult since reactive processes also come into play once the probe is presented (and vice versa). For instance, according to the DMC account, enhanced performance on AY-trials should be associated with an increased mobilization of reactive control in order to inhibit incorrect response tendencies associated with the A-cue. However, an enhanced performance on AY-trials could also result from a decreased utilization of proactive control mechanisms, resulting in a decreased response bias towards a target response and hence a decreased need for reactive mechanisms to resolve the competition at the response level (as also reflected by smaller N2 and P3a amplitudes). Similarly, a decreased performance on BX-trials, which the DMC account associated with reactive control mechanisms, could also be related to a decreased proactive control. More precisely, when the B-cue is not proactively maintained, it will activate a non-target response to a lesser extent. Consequently, when the X-probe is presented reactive control is not needed as much, as less response conflict is experienced. Thus, the X-probe will activate an erroneous targetresponse, resulting in decreased performance on BX-trials. Future studies using this task should be aware 
that while this task is able to examine the proactive-reactive control balance, it is less suited to completely disentangle proactive from reactive control.

Second, a shifted proactive-reactive control balance associated with trait rumination may be apparent only in individuals with higher levels of trait rumination. In our study, we used a sample of undergraduate students and volunteers and we controlled for clinical levels of depressive symptoms by excluding participants with elevated BDI-scores. To increase generalizability, we included individuals with a broad range of trait rumination scores (i.e., low, moderate and high levels of trait rumination). However, in previous studies investigating cognitive control in a sample of participants with a tendency to ruminate, the levels of trait rumination were higher compared to the ones in our sample (e.g., Beckwé et al., 2014; Hoorelbeke et al., 2015). For example, in a study of Beckwé et al. (2014), high ruminators were characterized by an RRS score $>56$ and the mean RRS score in this group was 65 . In a study of Hoorelbeke and colleagues (2015) increased levels of rumination were defined as an RRS score $\geq 43$ and the mean RRS scores in both groups were > 51. In our study, the mean RRS scores were 49 (range: 28 - 74) and 41 (range: 26 - 69), respectively for Experiments 1 and 2. Furthermore, previous studies composed their samples by only selecting participants with increased levels of trait rumination (RRS score $\geq 43$ ), which might also affect generalizability. Possibly, our results may underestimate the association between cognitive control and trait rumination, since we did not include those individuals with more elevated levels of trait rumination. Future studies should increase the range of trait rumination levels by including participants with lower as well as higher levels of trait rumination, which might improve the generalizability and reliability.

Third, the association between cognitive control and trait rumination might only be present in valenced situations. Previous studies investigating the link between proactive control and trait rumination (e.g., De Lissnyder et al., 2012; Zetsche et al., 2012) showed that proactive impairments in cognitive control in the context of rumination were mostly pronounced during the processing of negative information. For example, Zetsche et al. (2012) showed that a reduced ability to regulate and control negative material in WM predicted higher levels of trait rumination. Future studies should include tasks assessing proactive and reactive control using emotional (negative) stimulus material to investigate whether valence-specific control deficits are associated with trait rumination. Notably, due to the 
correlational nature of this study, we cannot draw any causal conclusions regarding the relationship between cognitive control and trait rumination. Further research should clarify the direction of the relationship between both constructs.

Fourth, the samples in our experiments were heavily skewed toward women and this could potentially bias the results. Future studies need to use more balanced samples. Fifth, in our study, we did not control for other psychopathologies related to rumination, except for depressive symptomatology. As we did not control for other psychopathology related to rumination, we were not able to measure the singular effect of cognitive control on trait rumination, without the interference of co-morbid mental disorders. Future studies should control for the mental health status of participants for example by means of a structural clinical interview assessing other possible co-morbid clinical disorders.

In conclusion, we investigated whether a shift towards more reactive control instead of proactive control, was associated with increased trait rumination using behavioral and electrophysiological measures. However, we found no evidence of such a disturbed proactive-reactive control balance to be associated with trait rumination. More research is needed to further investigate the proactive-reactive control balance in the context of trait rumination. 


\section{Acknowledgments}

We gratefully thank Lore Vandenhaute for her help with the study's data collection.

\section{Disclosure statement}

This work was supported by Grants of the Research Foundation Flanders, Belgium (FWOVlaanderen) awarded to Charlotte Muscarella (Grant Number: 11ZN315N and 11ZN317N). The authors have no financial interests or potential conflicts of interest to disclose. 


\section{References}

Aben, B., Calderon, C. B., Van der Cruyssen, L., Picksak, D., Van den Bussche, E., \& Verguts, T. (2019). Context-dependent modulation of cognitive control involves different temporal profiles of fronto-parietal activity. Neuroimage, 189, 755-762. http://dx.doi.org/10.1016/j.neuroimage.2019.02.004.

Aben, B., Calderon, C. B., Van den Bussche, E., \& Verguts, T. (2020). Cognitive effort modulates connectivity between dorsal anterior cingulate cortex and task-relevant cortical areas. Journal of Neuroscience, 40, 3838-3848. https://doi.org/10.1523/JNEUROSCI.2948-19.2020.

Alderman, B. L., Olson, R. L., Bates, M. E., Selby, E. A., Buckman, J. F., Brush, C. J. ... Shors, T. J. (2015). Rumination in major depressive disorder is associated with impaired neural activation during conflict monitoring. Frontiers in Human Neuroscience, 9, 269. http://dx.doi.org/10.3389/fnhum.2015.00269.

Altamirano, L. J., Miyake, A., \& Whitmer, A. J. (2010). When mental inflexibility facilitates executive control: Beneficial side effects of ruminative tendencies on goal maintenance. Psychological Science, 21, 1377-82. http://dx.doi.org/10.1177/0956797610381505.

Beck, A. T., Steer, R. A., \& Brown, G. K. (1996). Beck Depression Inventory: second edition manual. San Antonio (TX): The Psychological Corporation.

Beckwé, M., Deroost, N., Koster, E. H. W., De Lissnyder, E., \& De Raedt, R. (2014). Worrying and rumination are both associated with reduced cognitive control. Psychological Research, 78, 651660. http://dx.doi.org/10.1007/s00426-013-0517-5 
Beste, C., Domschke, K., Radenz, B., Falkenstein, M., \& Konrad, C. (2011). The functional 5-HT1A receptor polymorphism affects response inhibition processes in a context-dependent manner. Neuropsychologia, 49, 2664-2672. http://dx.doi.org/10.1016/j.neuropsychologia.2011.05.014.

Botvinick, M. M., Braver, T. S., Barch, D. M., Carter, C.S., \& Cohen, J. D. (2001). Conflict monitoring and cognitive control. Psychological Review, 108, 624-652. http://dx.doi.org/10.1037/0033-295X.108.3.624.

Braver, T. S. (2012). The variable nature of cognitive control: A dual mechanisms framework. Trends in Cognitive Science, 16, 106-13. http://dx.doi.org/10.1016/j.tics.2011.12.010.

Braver, T. S., \& Barch, D. M. (2002). A theory of cognitive control, aging cognition, and neuromodulation. Neuroscience \& Biobehavioral Reviews, 26, 809-17. http://dx.doi.org/10.1016/S0149-7634(02)00067-2.

Braver, T. S., \& Cohen, J. D. (2000). On the control of control: the role of dopamine in regulating prefrontal function and working memory. In: S. Monsell \& J. Driver (Eds). Attention and performance XVIII (pp. 713-38). Cambridge: MA: MIT Press.

Braver, T. S., \& Cohen, J. D. (2001). Working memory, cognitive control, and the prefrontal cortex: Computational and empirical studies. Cognitive Processing, 2, 25-55.

Braver, T. S., Gray, J. R., \& Burgess, G. C. (2007). Explaining the many varieties of working memory variation: Dual mechanisms of cognitive control. In: Conway, A., et al., editors. Variation in Working memory. Oxford University Press: Oxford. P79-106. 
Braver, T. S., Paxton, J. L., Locke, H. S., \& Barch, D. M. (2009). Flexible neural mechanisms of cognitive control within human prefrontal cortex. Proceedings of the National Academy of Sciences of the United States of America, 106, 7351-6. http://dx.doi.org/10.1073/pnas.0808187106.

Braver, T. S., Satpute, A. B., Rush, B. K., Racine, C. A., \& Barch, D. M. (2005). Context processing and context maintenance in healthy aging and early stage dementia of the Alzheimer's type. Psychology and Aging, 20, 33-46. http://dx.doi.org/10.1037/0882-7974.20.1.33.

Clayson, P. E., \& Larson, M. J. (2011). Conflict adaptation and sequential trial effects: Support for the conflict monitoring theory. Neuropsychologia, 49, 1953-1961. http://dx.doi.org/10.1016/j.neuropsychologia.2011.03.023.

Cohen, N., Mor, N., \& Henik, A. (2015). Linking executive control and emotional response: A training procedure to reduce rumination. Clinical Psychological Science, 3, 15-25. http://dx.doi.org/10.1177/2167702614530114.

Coles, M. G. H., Gratton, G., Bashore, T. R., Eriksen, C. W, \& Donchin, E. A. (1985). Psychophysiological investigation of the continuous flow model of human information processing. Journal of Experimental Psychology: Human Perception and Performance, 11, 529-561. http://dx.doi.org/10.1037/0096-1523.11.5.529.

Colzato, L. S, Steenbergen, L., \& Hommel, B. (2018). Rumination impairs the control of stimulusinduced retrieval of irrelevant information, but not attention, control, or response selection in general. Psychological Research. Advance online publication. http://dx.doi.org/10.1007/s00426018-0986-7. 
Davis, R. N., \& Nolen-Hoeksema, S. (2000). Cognitive inflexibility among ruminators and nonruminators. Cognitive Therapy and Research, 24, 699-711. http://dx.doi.org/10.1023/A:1005591412406.

Delaney, H. D., \& Maxwell, S. E. (1981). On using analysis of covariance in repeated measures designs. Multivariate Behavioral Research, 16, 105-23. http://dx.doi.org/10.1207/s15327906mbr1601_6.

De Lissnyder, E., Koster, E. H., \& De Raedt, R. (2012). Emotional interference in working memory is related to rumination. Cognitive Therapy and Research, 36, 348-357. http://dx.doi.org/10.1007/s10608-011-9352-4.

De Lissnyder, E., Koster, E. H., Goubert, L., Onraedt, T., Vanderhasselt, M. A., \& De Raedt, R. (2012). Cognitive control moderates the association between stress and rumination. Journal of Behavior Therapy and Experimental Psychiatry, 43, 519-25. http://dx.doi.org/10.1016/j.jbtep.2011.07.004.

Delorme, A., \& Makeig, S. (2004). EEGLAB: An open source toolbox for analysis of single-trial EEG dynamics including independent component analysis. Journal of Neuroscience Methods, 134, 9-21. http://dx.doi.org/10.1016/j.jneumeth.2003.10.009.

Demeyer, I., De Lissnyder, E., Koster, E. H. W., \& De Raedt, R. (2012). Rumination mediates the relationship between impaired cognitive control for emotional information and depressive symptoms: A prospective study in remitted depressed adults. Behaviour Research and Therapy, 50, 292-7. http://dx.doi.org/10.1016/j.brat.2012.02.012. 
Desender, K., Van Opstal, F., Hughes, G., \& Van den Bussche, E. (2016). The temporal dynamics of metacognition: Dissociating task-related activity from later metacognitive processes. Neuropsychologia, 82, 54-64. http://dx.doi.org/10.1016/j.neuropsychologia.2016.01.003.

Donchin, E., \& Coles, M. G. H. (1988). Is the P300 component a manifestation of context updating? Behavioral and Brain Sciences, 11, 357-74. http://dx.doi.org/10.1017/S0140525X00058027.

Edwards, B. G., Barch, D. M, \& Braver, T. S. (2010). Improving prefrontal cortex function in schizophrenia through focused training of cognitive control. Frontiers in Human Neuroscience, 4:32. http://dx.doi.org/10.3389/fnhum.2010.00032.

Eysenck, M. W., \& Calvo, M. G. (1992). Anxiety and performance: The processing efficiency theory. Cognition \& Emotion, 6, 409-434. https://doi.org/10.1080/02699939208409696

Eysenck, M. W., Derakshan, N., Santos, R., \& Calvo, M. G. (2007). Anxiety and cognitive performance: Attentional control theory. Emotion, 7, 336-353. http://dx.doi.org/10.1037/15283542.7.2.336.

Hanslmayr, S., Pastötter, B., Bäuml, K., Gruber, S., Wimber, M., \& Klimesch, W. (2008). The electrophysiological dynamics of interference during the stroop task. Journal of Cognitive Neuroscience, 20, 215-225. http://doi.org/10.1162/jocn.2008.20020.

Harvey, A. G., Watkins, E., Mansell, W., \& Shafran, R. (2004). Cognitive behavioural processes across psychological disorders: A transdiagnostic approach to research and treatment. Oxford: Oxford University Press.

Hoorelbeke, K., Koster, E. H. W., Demeyer, I., Loeys, L., \& Vanderhasselt, M. A. (2016). Effects of cognitive control training on the dynamics of (mal)adaptive emotion regulation in daily life. Emotion, 16, 945-956. http://dx.doi.org/10.1037/emo0000169. 
Hoorelbeke, K., Koster, E. H., Vanderhasselt, M. A., Callewaert, S., \& Demeyer, I. (2015). The influence of cognitive control training on stress reactivity and rumination in response to a lab stressor and naturalistic stress. Behaviour Research and Therapy, 69, 1-10. http://dx.doi.org/10.1016/j.brat.2015.03.010.

Jaspar, M., Manard, M., Dideberg, V., Bours, V., Maquet, P., \& Collette, F. (2016). Influence of COMT genotype on antero-posterior cortical functional connectivity underlying interference resolution. Cerebral Cortex, 26, 498-509. http://dx.doi.org/10.1093/cercor/bhu188.

Joormann, J. (2010). Cognitive inhibition and emotion regulation in depression. Current Directions in Psychological Science, 19, 161-166. http://dx.doi.org/10.1177/0963721410370293.

Joormann, J., \& D'Avanzato, C. (2010). Emotion regulation in depression: Examining the role of cognitive processes. Cognition and emotion, 24, 913-939. http://dx.doi.org/10.1080/02699931003784939.

Joormann, J., \& Gotlib, I. H. (2008). Updating the contents of working memory in depression: Interference from irrelevant negative material. Journal of Abnormal Psychology, 117, 182-92. http://dx.doi.org/10.1037/0021-843X.117.1.182.

Liotti, M., Woldorff, M.G., Perez, R., \& Mayberg, H.S. (2000). An ERP study of the temporal course of the Stroop color-word interference effect. Neuropsychologia, 38, 701-711. http://doi.org/10.1016/S0028-3932(99)00106-2.

Locke, H. S., \& Braver, T. S. (2008). Motivational influences on cognitive control: Behavior, brain activation, and individual differences. Cognitive, Affective, \& Behavioral Neuroscience, 8, 99-112. http://dx.doi.org/10.3758/CABN.8.1.99. 
Knight, R. T. (1984). Decreased response to novel stimuli after prefrontal lesions in man.

Electroencephalography and Clinical Neurophysiology, 59, 9-20. http://dx.doi.org/10.1016/01685597(84)90016-9.

Koster, E. H. W., De Lissnyder, E., Derakshan, N., \& De Raedt, R. (2011). Understanding depressive rumination from a cognitive science perspective: The impaired disengagement hypothesis. Clinical Psychology Review, 31, 138-145. http://dx.doi.org/10.1016/j.cpr.2010.08.005.

Krompinger, J. W., \& Simons, R. F. (2011). Cognitive inefficiency in depressive undergraduates: Stroop processing and ERPs. Biological Psychologyl, 86, 239-46. http://dx.doi.org/10.1016/j.biopsycho.2010.12.004.

Miller, E. K., \& Cohen, J. D. (2001). An integrative theory of prefrontal cortex function. Annual Review of Neuroscience, 24, 167-202. http://dx.doi.org/10.1146/annurev.neuro.24.1.167.

Miyake, A., Friedman, N., Emerson, M., Witzki, A., Howerter, A., \& Wager, T. (2000). The Unity and Diversity of Executive Functions and Their Contributions to Complex "Frontal Lobe" Tasks: A Latent Variable Analysis. Cognitive Psychology, 41, 49-100. http://doi.org/10.1006/cogp.1999.0734

Morales, J., Yudes, C., Gómez-Ariza, C. J., \& Bajo, M. T. (2015). Bilingualism modulates dual mechanisms of cognitive control: Evidence from ERPs. Neuropsychologia, 66, 157-69. http://dx.doi.org/10.1016/j.neuropsychologia.2014.11.014.

Muscarella, C., Mairesse, O., Hughes, G., Neu, D., \& Van den Bussche, E. (2019). Recruitment dynamics of cognitive control in insomnia. Sleep, 42, 1-19. http://dx.doi.org/10.1093/sleep/zsz039. 
Nee, D. E., Wager, T. D., \& Jonides, J. (2007). Interference resolution: Insights from a meta-analysis of neuroimaging tasks. Cognitive, Affective and Behavioral Neuroscience, 7, 1-17. http://dx.doi.org/10.3758/CABN.7.1.1.

Nolen-Hoeksema, S., Wisco, B. E., \& Lyubomirsky, S. (2008). Rethinking rumination. Perspectives on Psychological Science, 3, 400-24. http://dx.doi.org/10.1111/j.1745-6924.2008.00088.x.

Paxton, J. L., Barch, D. M., Racine, C. A., \& Braver, T. S. (2008). Cognitive control, goal maintenance, and prefrontal function in healthy aging. Cerebral Cortex, 18, 1010-1028. http://dx.doi.org/10.1093/cercor/bhm135.

Polich, J. (2007). Updating P300: An integrative theory of P3a and P3b. Clin Neurophysiol, 118, 2128-48. http://dx.doi.org/10.1016/j.clinph.2007.04.019.

Polich, J., \& Comerchero, M. D. (2003). P3a from visual stimuli: Typicality, task, and topography. Brain Topography, 15, 141-52. http://dx.doi.org/10.1023/A:1022637732495.

Purmann, S., Badde, S., Luna-Rodriguez, A., \& Wendt, M. (2011). Adaptation to frequent conflict in the eriksen flanker task. Journal of Psychophysiology, 25, 50-59. http://dx.doi.org/10.1027/0269$8803 / \mathrm{a} 000041$.

Raes, F., Schoofs, H., Hoes, D., Hermans, D., Van Den Eede, F., \& Franck, E. (2009). 'Reflection’ en 'brooding' als subtypes van rumineren: Een herziening van de Ruminative response scale ['Reflection' and 'brooding' as subtypes of rumination: A revision of the Ruminative response scale]. Gedragstherapie, 42, 205-214. 
Rosvold, H. E., Mirsky, A. F., Sarason, I., \& Bransome, E. D. Jr., \& Beck, L. H. (1956). A continuous performance test of brain damage. Journal of Consulting and Clinical Psychology, 20, 343-350. http://dx.doi.org/10.1037/h0043220 1956.

Schoofs, H., Hermans, D., \& Raes, F. (2010). Brooding and reflection as subtypes of rumination: Evidence from confirmatory factor analysis in nonclinical samples using the dutch Ruminative response scale. Journal of Psychopathology and Behavioral Assessment, 32, 609-617. http://dx.doi.org/10.1007/s10862-010-9182-9.

Schupp, H. T., Lutzenberger, W., Rau, H., \& Birbaumer, N. (1994). Positive shifts of event related potentials: A state of cortical disfacilitation as reflected by the startle reflex probe. Electroencephalography and Clinical Neurophysiology, 90, 135-144. http://dx.doi.org/10.1016/0013-4694(94)90005-1.

Servan-Schreiber, D., Cohen, J. D., \& Steingard, S. (1996). Schizophrenic deficits in the processing of context. A test of a theoretical model. Archives of General Psychiatry, 53, 1105-1112. http://dx.doi.org/10.1001/archpsyc.1996.01830120037008.

Siemer, M. (2005). Mood-congruent cognitions constitute mood experience. Emotion, 5, 296-308. https://doi.org/10.1037/1528-3542.5.3.296

Smith, J. L., Johnstone, S. J., \& Barry, R. J. (2008). Movement-related potentials in the Go/NoGo task: The P3 reflects both cognitive and motor inhibition. Clinical Neurophysiology, 119, 704-714. http://dx.doi.org/10.1016/j.clinph.2007.11.042.

Treynor, W., Gonzalez, R., \& Nolen-Hoeksema, S. (2003). Rumination reconsidered: A psychometric analysis. Cognitive Therapy and Research, 27, 247-259. http://dx.doi.org/10.1023/A:1023910315561. 
Van der Does, A. J. W. (2002). Handleiding: De Nederlandse versie van de Beck Depression Inventory, 2de Editie (The Dutch version of the Beck Depression Inventory, 2nd Edition). Lisse: Swetsand Zeitlinger b.v.

Van Veen, V., \& Carter, C. S. (2002). The timing of action-monitoring processes in the anterior cingulate cortex. Journal of Cognitive Neuroscience, 14, 593-602. http://dx.doi.org/10.1162/08989290260045837.

van Wouwe, N. C., Band, G. P., \& Ridderinkhof, K. R. (2011). Positive affect modulates flexibility and evaluative control. Journal of Cognitive Neuroscience, 23, 524-39. http://dx.doi.org/10.1162/jocn.2009.21380.

Walter, W. G., Cooper, R., Aldridge, V. J., Mccallum, W. C., \& Winter, A. L. (1964). Contingent negative variation: An electric sign of sensorimotor association and expectancy in the human brain. Nature, 203, 380-384. http://dx.doi.org/10.1038/203380a0.

Watkins, E. R. (2008). Constructive and unconstructive repetitive thought. Psychological Bulletin, 134, 163-206. http://dx.doi.org/10.1037/0033-2909.134.2.163.

West, R., \& Alain, C. (2000). Effects of task context and fluctuations of attention on neural activity supporting performance of the stroop task. Brain Research, 873, 102-11. http://dx.doi.org/10.1016/S0006-8993(00)02530-0.

Whitmer, A. J., \& Banich, M. T. (2007). Inhibition versus switching deficits in different forms of rumination. Psychol Sci,18(6), 546-53. http://dx.doi.org/10.1111/j.1467-9280.2007.01936.x. 
Whitmer, A. J., \& Gotlib, I. H. (2013). An attentional scope model of rumination. Psychological Bulletin, 139, 1036-61. http://dx.doi.org/10.1037/a0030923.

Yang, Y., Cao, S., Shields, G. S., Teng, Z., \& Liu, Y. (2017). The relationships between rumination and core executive functions: A meta-analysis. Depression and Anxiety, 34, 37-50. http://dx.doi.org/10.1002/da.22539.

Yoon, K. L., LeMoult, J., Joormann, J. (2014). Updating emotional content in working memory: A depression-specific deficit? Journal of Behavior Therapy and Experimental Psychiatry, 45, 368374. http://dx.doi.org/10.1016/j.jbtep.2014.03.004.

Zetsche, U., Bürkner, P. C, \& Schulze, L. (2018). Shedding light on the association between repetitive negative thinking and deficits in cognitive control - A meta-analysis. Clinical Psychology Review, 63, 56-65. http://dx.doi.org/10.1016/j.cpr.2018.06.001.

Zetsche, U., D’Avanzato, C., \& Joormann, J. (2012). Depression and rumination: relation to components of inhibition. Cognition and Emotion, 26, 758-67. http://dx.doi.org/10.1080/02699931.2011.613919.

Zetsche, U., \& Joormann, J. (2011). Components of interference control predict depressive symptoms and rumination cross-sectionally and at six months follow-up. Journal of Behavior Therapy and Experimental Psychiatry, 42, 65-73. http://dx.doi.org/10.1016/j.jbtep.2010.06.001. 